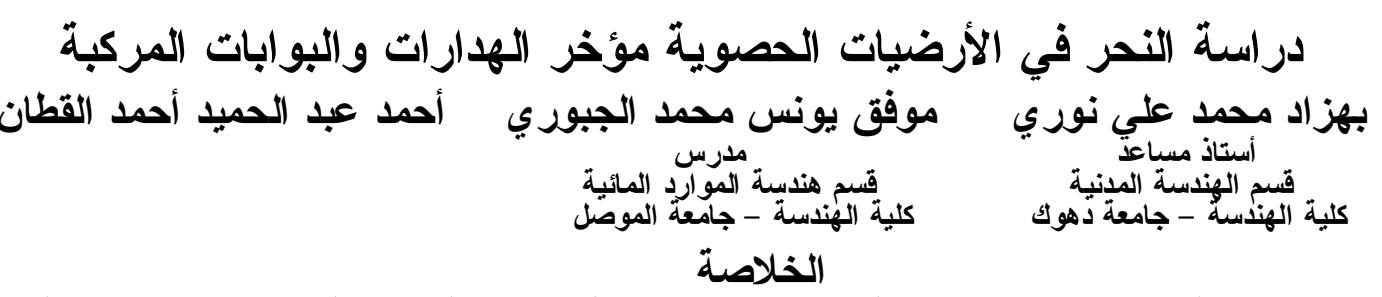

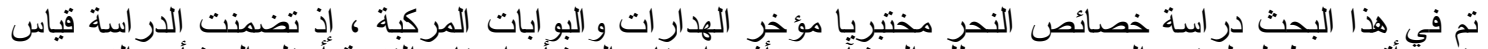

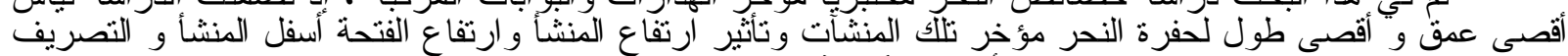

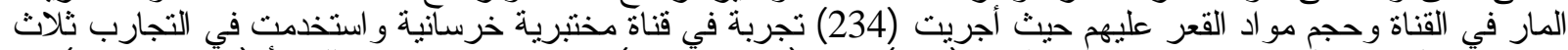

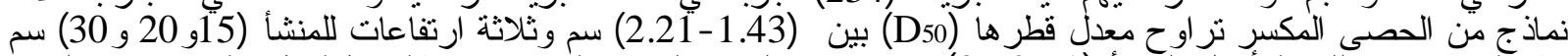

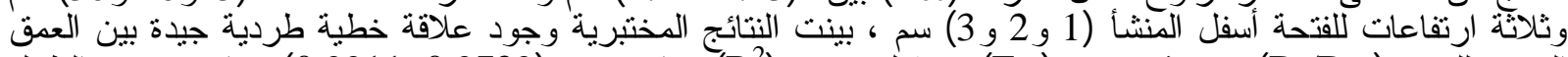

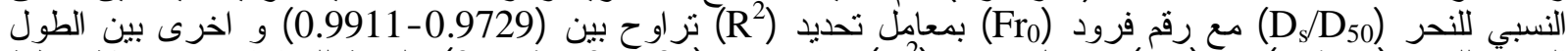
النسبي للحر

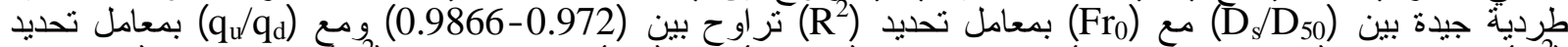

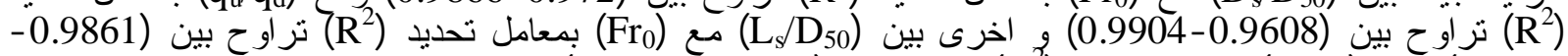

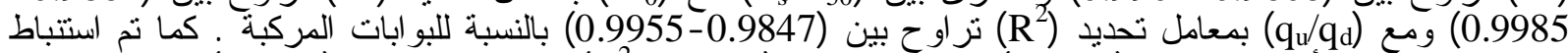

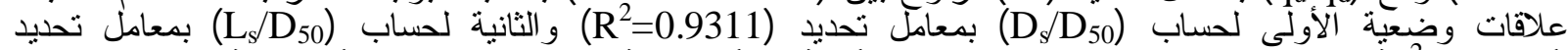

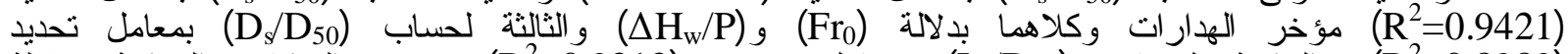

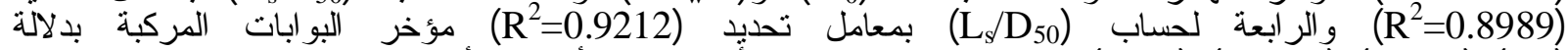
و

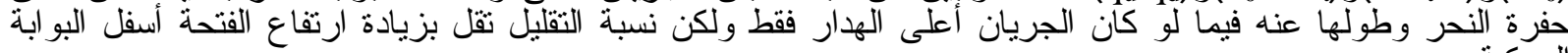

\title{
Laboratory Comparison Study of Scour Downstream Weirs and Communed Gates
}

\author{
Bahzad M. A. Noori \\ Asst. Prof. \\ Dept. of Civil Eng. \\ Coll. of Eng. / Univ. of Duhok
}

\section{Mwafaq Y. M. Aljubori Lect.}

Dept. of Water Recourses Eng.

Coll. of Eng. / Univ. of Mosul

\section{Ahmad A. A. Alkatan}

\section{Abstract}

In the present investigation, a laboratory study of scour characteristics downstream weirs , compound gates has been conducted. The study included the measurement of maximum scour depth and the length of scour hole downstream these structures. Also, the effects of structure height, undersluice opening height, discharge variation and bed material size on the depth and length of scour hole, Two hundred eighty eight Experiments were conducted in a concrete laboratory channel Three sizes of rocks were used are in range of $(1.43-2.21) \mathrm{cm}$ and three of 15,20 and $30 \mathrm{~cm}$ and Three under gate opening heights of 1,2 and $3 \mathrm{~cm}$ were tested Experimental. Results of this study showed a good direct relationships between relative scour depth $\left(\mathrm{D}_{\mathrm{S}} / \mathrm{D}_{50}\right)$ with Froud Number $\left(\mathrm{Fr}_{\mathrm{o}}\right)$ of correlation factor $\left(\mathrm{R}^{2}\right)$ between $(0.9729-0.9911)$ and other between relative scour length $\left(\mathrm{L}_{\mathrm{g}} / \mathrm{D}_{50}\right)$ with $\left(\mathrm{Fr}_{\mathrm{o}}\right)$ of correlation factor $\left(\mathrm{R}^{2}\right)$ between $(0.9636-0.9947)$ for weirs and another good direct relationships between $\left(\mathrm{D}_{s} / \mathrm{D}_{50}\right)$ with $\left(\mathrm{Fr}_{0}\right)$ of correlation factor $\left(\mathrm{R}^{2}\right)$ between $(0.972-0.9866)$ and with $\left(\mathrm{q}_{\mathrm{u}} / \mathrm{q}_{\mathrm{d}}\right)$ of correlation factor $\left(\mathrm{R}^{2}\right)$ between $(0.9608-0.9904)$ and other relationship between $\left(\mathrm{L}_{s} / \mathrm{D}_{50}\right)$ with $\left(\mathrm{Fr}_{\mathrm{o}}\right)$ of correlation factor $\left(\mathrm{R}^{2}\right)$ between $(0.9861-0.9985)$ and with $\left(\mathrm{q}_{\mathrm{u}} / \mathrm{q}_{\mathrm{d}}\right)$ of correlation factor $\left(\mathrm{R}^{2}\right)$ between $(0.9847-0.9955)$, many relationships were obtained the first to estimate $\left(\mathrm{D}_{\mathrm{S}} / \mathrm{D}_{50}\right)$ of correlation factor $\left(\mathrm{R}^{2}=0.9311\right)$, the second for $\left(\mathrm{L}_{\mathrm{S}} / \mathrm{D}_{50}\right)$ of correlation factor $\left(\mathrm{R}^{2}=0.9421\right)$ downstream weirs each of them in terms of $\left(\mathrm{Fr}_{0}\right)$ and $\left(\Delta \mathrm{H}_{\mathrm{w}} / \mathrm{P}\right)$, the third to estimate $\left(\mathrm{D}_{s} / \mathrm{D}_{50}\right)$ of correlation factor $\left(\mathrm{R}^{2}=0.8989\right)$ and the forth for $\left(\mathrm{L}_{\mathrm{s}} / \mathrm{D}_{50}\right)$ of correlation factor $\left(\mathrm{R}^{2}=0.9212\right)$ downstream compound gates each of them in terms of $\left(\mathrm{Fr}_{0}\right),\left(\Delta \mathrm{H}_{\mathrm{w}} / \mathrm{P}\right),\left(\mathrm{h}_{0} / \Delta \mathrm{H}_{\mathrm{t}}\right)$ and $\left(\mathrm{q}_{\mathrm{u}} / \mathrm{q}_{\mathrm{d}}\right)$. It was also shown that the scour depth and length downstream compound gates are less than those downstream weirs but the percentage decrement will decrease if the opening of compound gate is increased .

Key Words : Hydraulic, Scour, Scour Downstream Weirs and Compound Gates . 
Al-Rafidain Engineering Vol.17 No.5

October 2009

\begin{tabular}{|c|c|c|c|c|c|}
\hline \multicolumn{6}{|c|}{ قائمة الرموز } \\
\hline التسمية & الرمز & التسمية & الرمز & التسمية & الرمز \\
\hline القناة: النسبة & $Y_{n}$ & المنثقأ . الماء فوق عافة & $\mathrm{h}^{\prime}$ & معاملات. & $\begin{array}{c}a_{1}, b_{1}, a_{2}, b_{2,} a_{3} \\
, b_{3}, a_{4}, b_{4}, a_{5} \\
b_{5}, a_{6}, b_{6}\end{array}$ \\
\hline 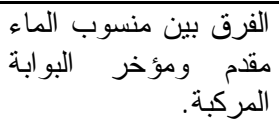 & $\Delta \mathrm{H}_{\mathrm{t}}$ & البوابة المركبة . . الفتحة السفلية & $\mathrm{h}_{0}$ & ثلى إلى النسبة بين المعادلة (Eggenberger) وتصريف المار فوق البوابة قيمته & C \\
\hline مقام ومؤخر الهدار . الماء & $\Delta \mathrm{H}_{\mathrm{w}}$ & ارتقاع المنشأ. & P & عمق حفرة النحر . & $D_{s}$ \\
\hline المواد القعر والماء . الكتلية & $\Delta \rho_{\mathrm{s}}$ & العرض التصريف المار أعلى وأسفل الوحلة & $\mathrm{q}_{\mathrm{t}}$ & متوسط قطر مو اد القعر . & $\mathrm{D}_{50}$ \\
\hline الكثافة الكتلية للماء. & $\rho_{\mathrm{w}}$ & التصريف لوحلى البو العرابة المركبة. & $\mathrm{q}_{\mathrm{u}}$ & قطعر فتحة الدنخل التي تمرر 90\% من مواد & ${ }_{09} \mathrm{D}$ \\
\hline اللزوجة الديناميكية. & $\mu$ & التصريف أسفل البو ابة المركبة. العرض. & $\mathrm{q}_{\mathrm{d}}$ & رقم فرود بدلالة كثافة مو اد القعر . & $\mathrm{Fr}_{0}$ \\
\hline & & المار فوق الهدار. لوحدة العرض & $q_{w}$ & عمق الجريان مؤخر البو ابة المركبة. & $\mathrm{h}_{2 \mathrm{t}}$ \\
\hline & & رقم رينولدز . & $\operatorname{Re}$ & عمق الجريان مؤخر الهدارات. & $\mathrm{h}_{2 \mathrm{w}}$ \\
\hline
\end{tabular}

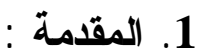

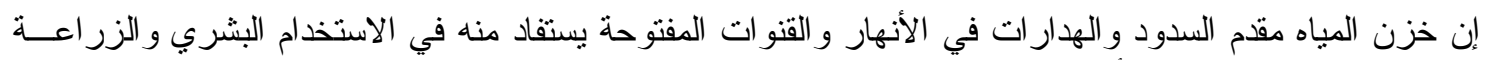

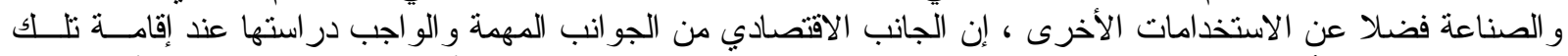

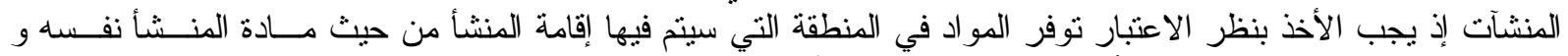

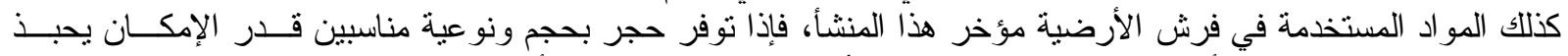

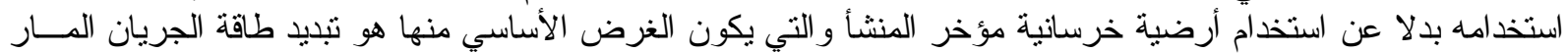

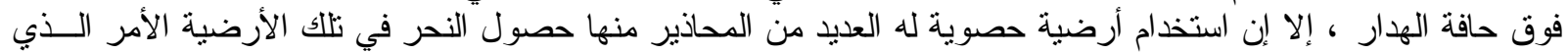

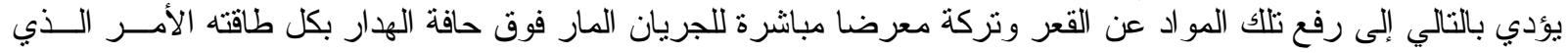

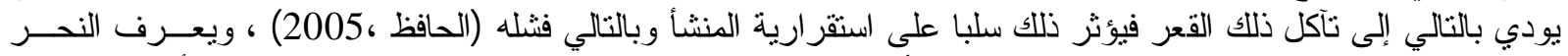

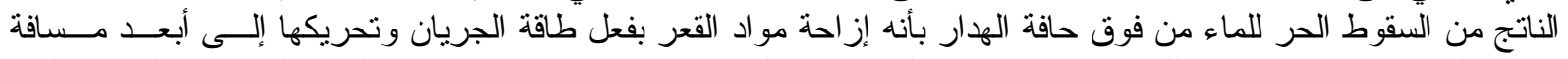

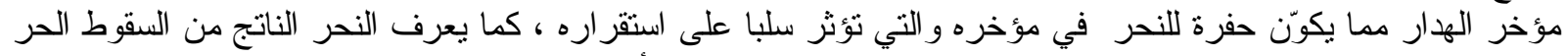

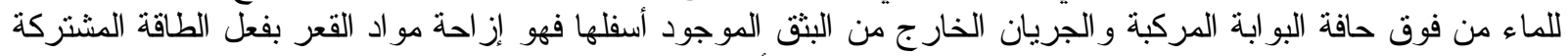

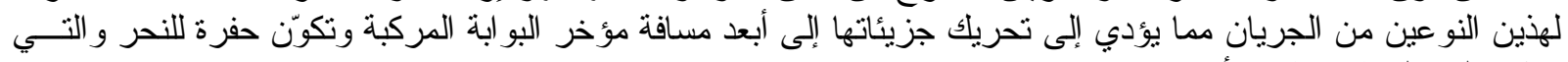
تؤُثر سلبا على استقر ار ها أيضا.

\section{2. عرض البحوث السابقة : مئة}

لقد عنيت مشكلة النحر مؤخر المنشآت الهيدروليكية كالهارار ات و المساقط المائية و السدود و غير ها ومنذ القدم

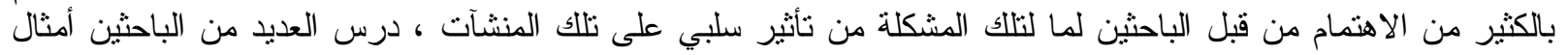

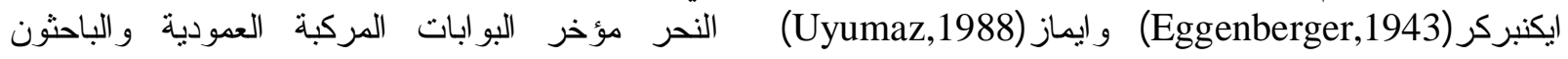

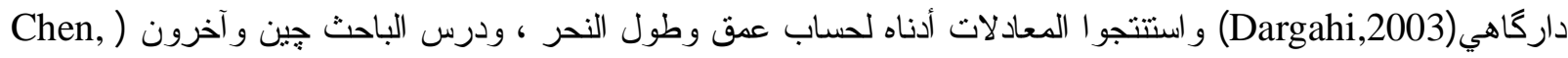

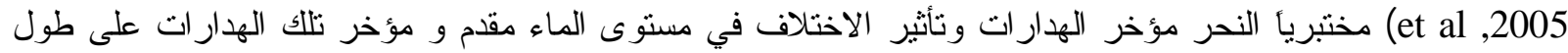

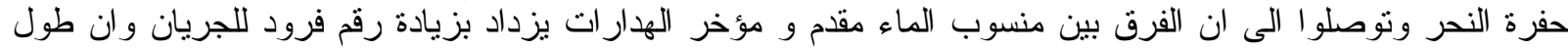

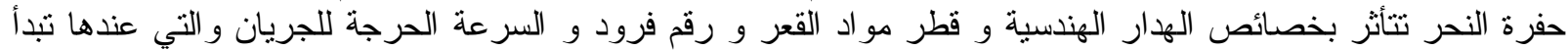




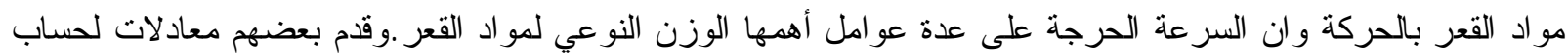

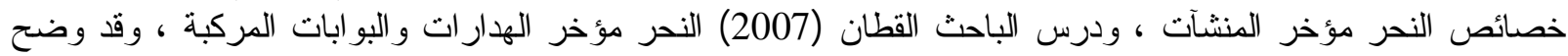
(Simons and Şentürk,1992)

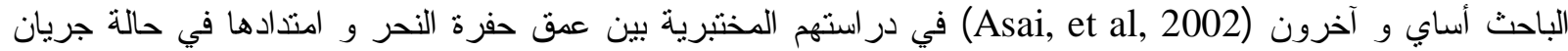

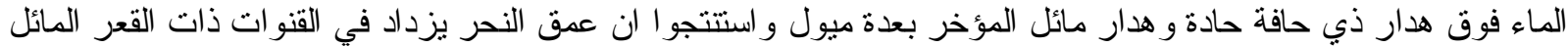

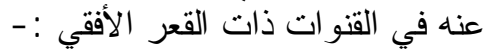

$D_{s}=C\left(\frac{\Delta H_{t}^{0.2} q_{t}^{0.6}}{D_{90}^{0.4}}\right)-h_{2 t}$

Eggenberger (Dargahi,2003 مذكور في )

$D_{s}=w \frac{\Delta H_{t}^{0.5} q_{t}^{0.6}}{D 90^{0.4}}$

Uyumaz

$\frac{\boldsymbol{D}_{s}}{\Delta \boldsymbol{H}_{w}}=\left(\frac{\Delta \boldsymbol{H}_{w}}{\boldsymbol{D}_{50}}\right)^{a}$

Dargahi

$\frac{\boldsymbol{L}_{s}}{\Delta \boldsymbol{H}_{w}}=\left(\frac{\Delta \boldsymbol{H}_{w}}{D_{50}}\right)^{b}$

\section{Dargahi}

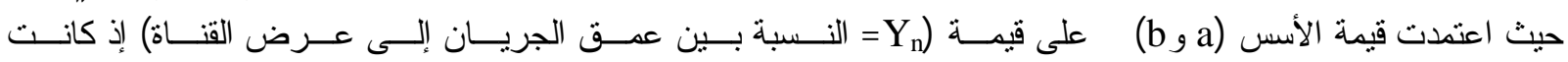
. لقيمة (a=1/3,b=1/4.5)

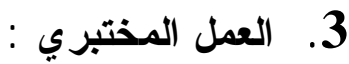

أجريت التجارب في قناة مشيدة من الخرسانة بطول (24.64 م) و بعرض (0.81 م) و بعمق (0.76 م) ، ثبتـت

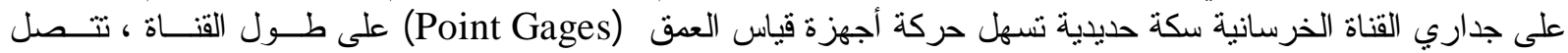

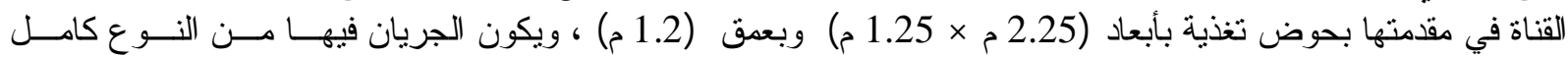

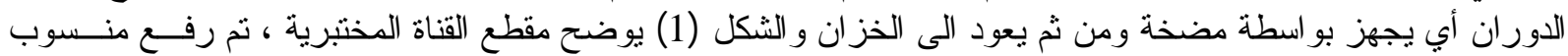

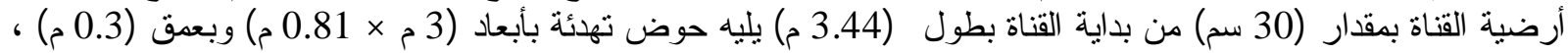

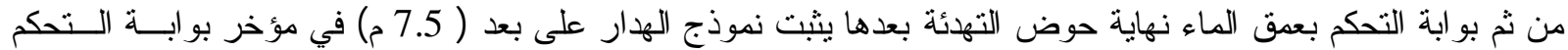

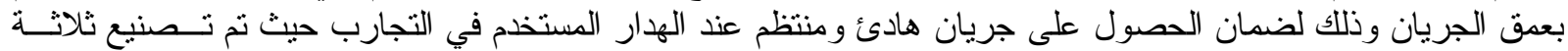

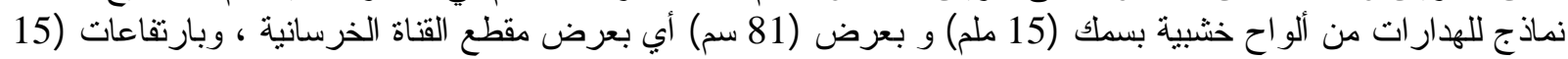

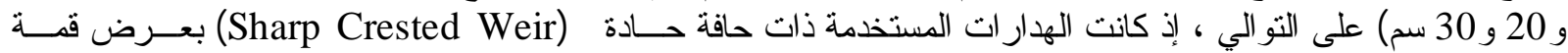

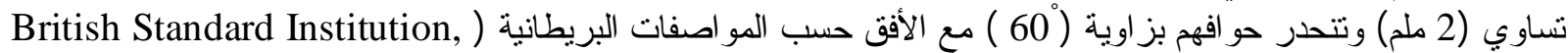

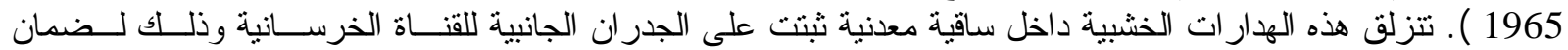

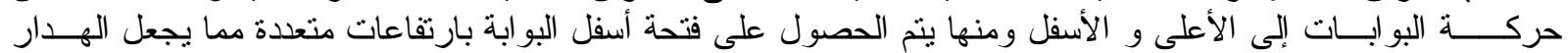

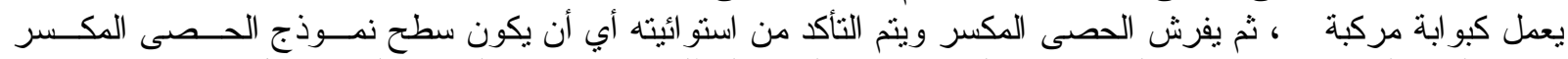

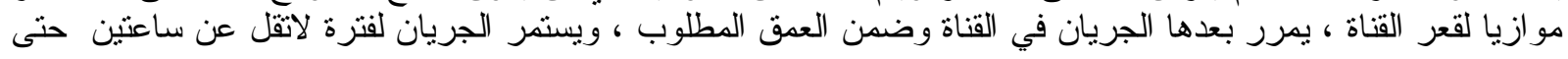

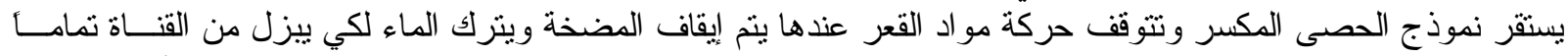

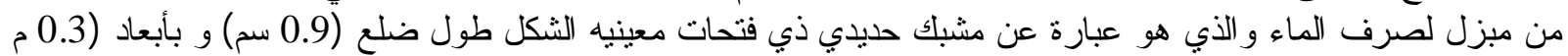

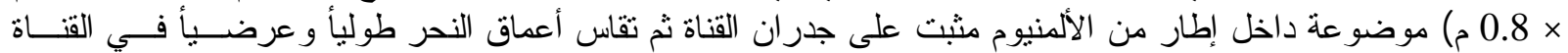

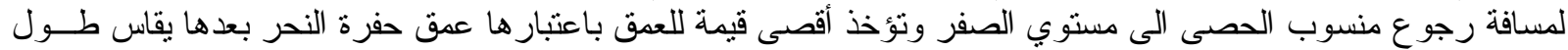

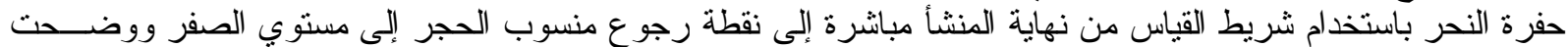

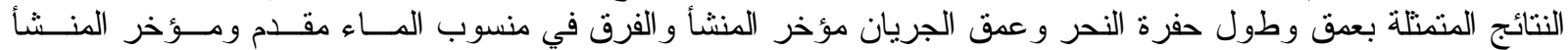

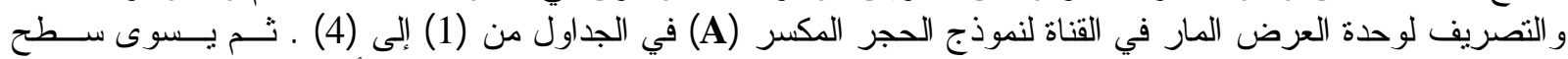

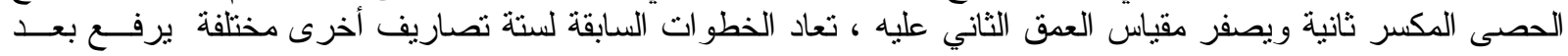




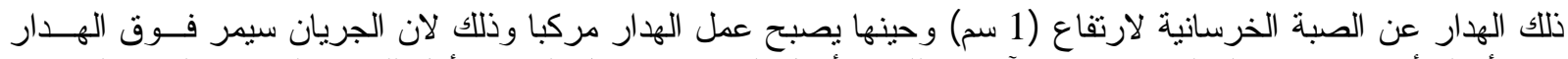

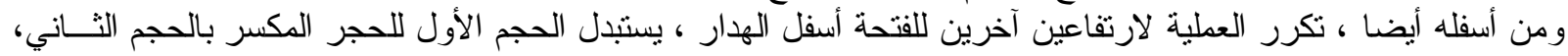
ومن ثم بالحجم الثالث ، ويوضح ، ولكر الجدول لإنفاعن (5) برنامج التجارب المختبرية .

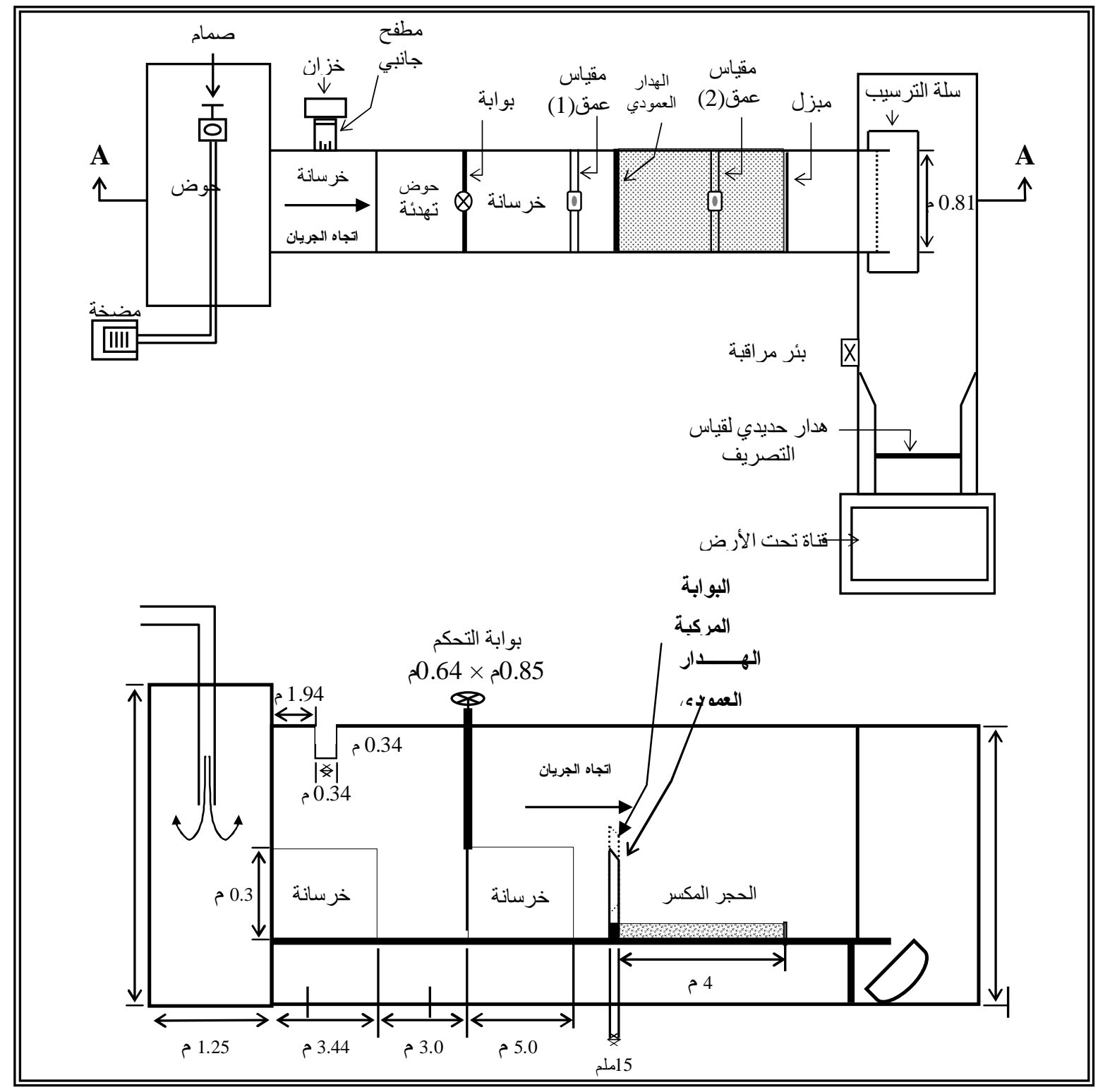

الثكل (1) : القتاة المختبرية التي أجريت فيها التجارب 
نوري : دراسة النحر في الأرضيات الحصوية مؤخر الهدارات والبوابات المركبة

جدول (1) : قياسات تجارب الهـار بالارتفاع الأول (P) مع النموذج (A)

\begin{tabular}{|c|c|c|c|c|c|}
\hline $\begin{array}{c}\mathbf{h}^{`} \\
(\mathrm{~cm})\end{array}$ & $\begin{array}{c}\mathbf{D}_{\mathbf{s}} \\
(\mathrm{cm})\end{array}$ & $\begin{array}{c}\mathbf{L}_{\mathbf{s}} \\
(\mathrm{cm})\end{array}$ & $\begin{array}{c}\mathbf{h}_{\mathbf{2 w}} \\
(\mathrm{cm})\end{array}$ & $\begin{array}{c}\Delta \mathbf{H}_{\mathbf{w}} \\
(\mathrm{cm})\end{array}$ & $\begin{array}{c}\mathbf{q}_{\mathbf{w}} \\
\left(\mathrm{m}^{3} / \mathrm{sec} / \mathrm{m}\right)\end{array}$ \\
\hline $\mathbf{2}$ & 4.2 & 14.8 & 3.00 & 14.00 & 0.0295 \\
\hline $\mathbf{3}$ & 4.4 & 16.1 & 0.83 & 14.20 & 0.0348 \\
\hline $\mathbf{4}$ & 6.7 & 20.1 & 0.54 & 14.50 & 0.0435 \\
\hline $\mathbf{5}$ & 8.2 & 24.8 & 0.15 & 14.90 & 0.0519 \\
\hline $\mathbf{6}$ & 9.8 & 28.7 & 09.5 & 15.10 & 0.0617 \\
\hline $\mathbf{7}$ & 11.5 & 34.3 & 06.1 & 15.90 & 0.0712 \\
\hline $\mathbf{8}$ & 12.5 & 39.0 & 07.6 & 16.30 & 0.0795 \\
\hline
\end{tabular}

جدول (2) : قياسات تجارب البوابة المركبة بالارتفاع الأول والفتحة الأولى (V (A) مع النموذج (A)

\begin{tabular}{|c|c|c|c|c|c|c|c|}
\hline $\begin{array}{c}\mathbf{h}^{`} \\
(\mathrm{~cm})\end{array}$ & $\begin{array}{c}\mathbf{D}_{\mathbf{s}} \\
(\mathrm{cm})\end{array}$ & $\begin{array}{c}\mathbf{L}_{\mathbf{s}} \\
(\mathrm{cm})\end{array}$ & $\begin{array}{c}\mathbf{h}_{\mathbf{2 t}} \\
(\mathrm{cm})\end{array}$ & $\begin{array}{c}\Delta \mathbf{H}_{\mathbf{t}} \\
(\mathrm{cm})\end{array}$ & $\begin{array}{c}\mathbf{\mathbf { q } _ { \mathbf { u } }} \\
\left(\mathrm{m}^{3} / \mathrm{sec} / \mathrm{m}\right)\end{array}$ & $\begin{array}{c}\mathbf{q}_{\mathbf{d}} \\
\left(\mathrm{m}^{3} / \mathrm{sec} / \mathrm{m}\right)\end{array}$ & $\begin{array}{c}\mathbf{q}_{\mathbf{t}} \\
\left(\mathrm{m}^{3} / \mathrm{sec} / \mathrm{m}\right)\end{array}$ \\
\hline $\mathbf{2}$ & 3.9 & 16.1 & 6.10 & 11.90 & 0.0056 & 0.0435 & 0.0491 \\
\hline $\mathbf{3}$ & 4.1 & 17.8 & 7.00 & 12.00 & 0.0101 & 0.0431 & 0.0531 \\
\hline $\mathbf{4}$ & 5.8 & 22.2 & 7.90 & 12.10 & 0.0154 & 0.0443 & 0.0597 \\
\hline $\mathbf{5}$ & 6.7 & 28.1 & 8.90 & 12.10 & 0.0215 & 0.0464 & 0.0679 \\
\hline $\mathbf{6}$ & 8.1 & 31.7 & 9.70 & 12.20 & 0.0283 & 0.0479 & 0.0762 \\
\hline $\mathbf{7}$ & 9.0 & 34.0 & 10.80 & 12.30 & 0.0358 & 0.0491 & 0.0849 \\
\hline $\mathbf{8}$ & 10.2 & 40.2 & 11.60 & 12.40 & 0.0440 & 0.0510 & 0.0950 \\
\hline
\end{tabular}

جدول (3) : قياسات تجارب البوابة المركبة بالارتفاع الأول و الفتحة الثانية (V) (A) مع النموذج (1)

\begin{tabular}{|c|c|c|c|c|c|c|c|}
\hline $\begin{array}{c}\mathbf{h}^{\prime} \\
(\mathrm{cm})\end{array}$ & $\begin{array}{c}\mathbf{D}_{\mathbf{s}} \\
(\mathrm{cm})\end{array}$ & $\begin{array}{c}\mathbf{L}_{\mathbf{s}} \\
(\mathrm{cm})\end{array}$ & $\begin{array}{c}\mathbf{h}_{2 \mathbf{t}} \\
(\mathrm{cm})\end{array}$ & $\begin{array}{c}\Delta \mathbf{H}_{\mathrm{t}} \\
(\mathrm{cm})\end{array}$ & $\underset{\left(\mathrm{m}^{3} / \mathrm{sec} / \mathrm{m}\right)}{\mathbf{q}_{\mathbf{u}}}$ & $\underset{\left(\mathrm{m}^{3} / \mathrm{sec} / \mathrm{m}\right)}{\mathbf{q}_{\mathbf{d}}}$ & $\underset{\left(\mathrm{m}^{3} / \mathrm{sec} / \mathrm{m}\right)}{\mathbf{q}_{\mathbf{t}}}$ \\
\hline 2 & 3.7 & 17.0 & 7.00 & 12.00 & 0.0056 & 0.0527 & 0.0583 \\
\hline 3 & 4.0 & 19.2 & 8.00 & 12.00 & 0.0100 & 0.0531 & 0.0631 \\
\hline 4 & 5.1 & 25.1 & 8.90 & 12.10 & 0.0153 & 0.0552 & 0.0705 \\
\hline 5 & 6.1 & 29.0 & 9.70 & 12.30 & 0.0214 & 0.0569 & 0.0783 \\
\hline 6 & 6.9 & 33.2 & 10.50 & 12.50 & 0.0282 & 0.0576 & 0.0858 \\
\hline 7 & 8.2 & 35.1 & 11.50 & 12.50 & 0.0357 & 0.0586 & 0.0943 \\
\hline 8 & 9.4 & 41.5 & 12.10 & 12.90 & 0.0438 & 0.0580 & 0.1018 \\
\hline
\end{tabular}

جدول (4) : قياسات تجارب البوابة المركبة بالارتفاع الأول و الفتحة الثالثة (V) (A) مع النموذج (A)

\begin{tabular}{|c|c|c|c|c|c|c|c|}
\hline $\begin{array}{c}\mathbf{h}^{\prime} \\
(\mathrm{cm})\end{array}$ & $\begin{array}{c}\mathbf{D}_{\mathbf{s}} \\
(\mathrm{cm})\end{array}$ & $\begin{array}{c}\mathbf{L}_{\mathbf{s}} \\
(\mathrm{cm})\end{array}$ & $\begin{array}{c}\mathbf{h}_{\mathbf{2 t}} \\
(\mathrm{cm})\end{array}$ & $\begin{array}{c}\Delta \mathbf{H}_{\mathbf{t}} \\
(\mathrm{cm})\end{array}$ & $\begin{array}{c}\mathbf{q}_{\mathbf{u}} \\
\left(\mathrm{m}^{3} / \mathrm{sec} / \mathrm{m}\right)\end{array}$ & $\begin{array}{c}\mathbf{q}_{\mathbf{d}} \\
\left(\mathrm{m}^{3} / \mathrm{sec} / \mathrm{m}\right)\end{array}$ & $\begin{array}{c}\mathbf{q}_{\mathbf{t}} \\
\left(\mathrm{m}^{3} / \mathrm{sec} / \mathrm{m}\right)\end{array}$ \\
\hline $\mathbf{2}$ & 6.6 & 15.8 & 9.30 & 10.70 & 0.0056 & 0.0690 & 0.0746 \\
\hline $\mathbf{3}$ & 7.1 & 23.3 & 9.90 & 11.10 & 0.0100 & 0.0708 & 0.0808 \\
\hline $\mathbf{4}$ & 7.9 & 25.0 & 10.30 & 11.70 & 0.0153 & 0.0722 & 0.0875 \\
\hline $\mathbf{5}$ & 8.0 & 28.5 & 11.40 & 11.60 & 0.0214 & 0.0727 & 0.0941 \\
\hline $\mathbf{6}$ & 9.1 & 32.8 & 12.00 & 12.00 & 0.0282 & 0.0724 & 0.1006 \\
\hline $\mathbf{7}$ & 10.2 & 40.0 & 12.70 & 12.30 & 0.0356 & 0.0737 & 0.1093 \\
\hline $\mathbf{8}$ & 11.9 & 46.3 & 13.60 & 12.40 & 0.0437 & 0.0751 & 0.1188 \\
\hline
\end{tabular}




\begin{tabular}{llll} 
Al-Rafidain Engineering & Vol.17 & No.5 & October 2009 \\
\hline
\end{tabular}

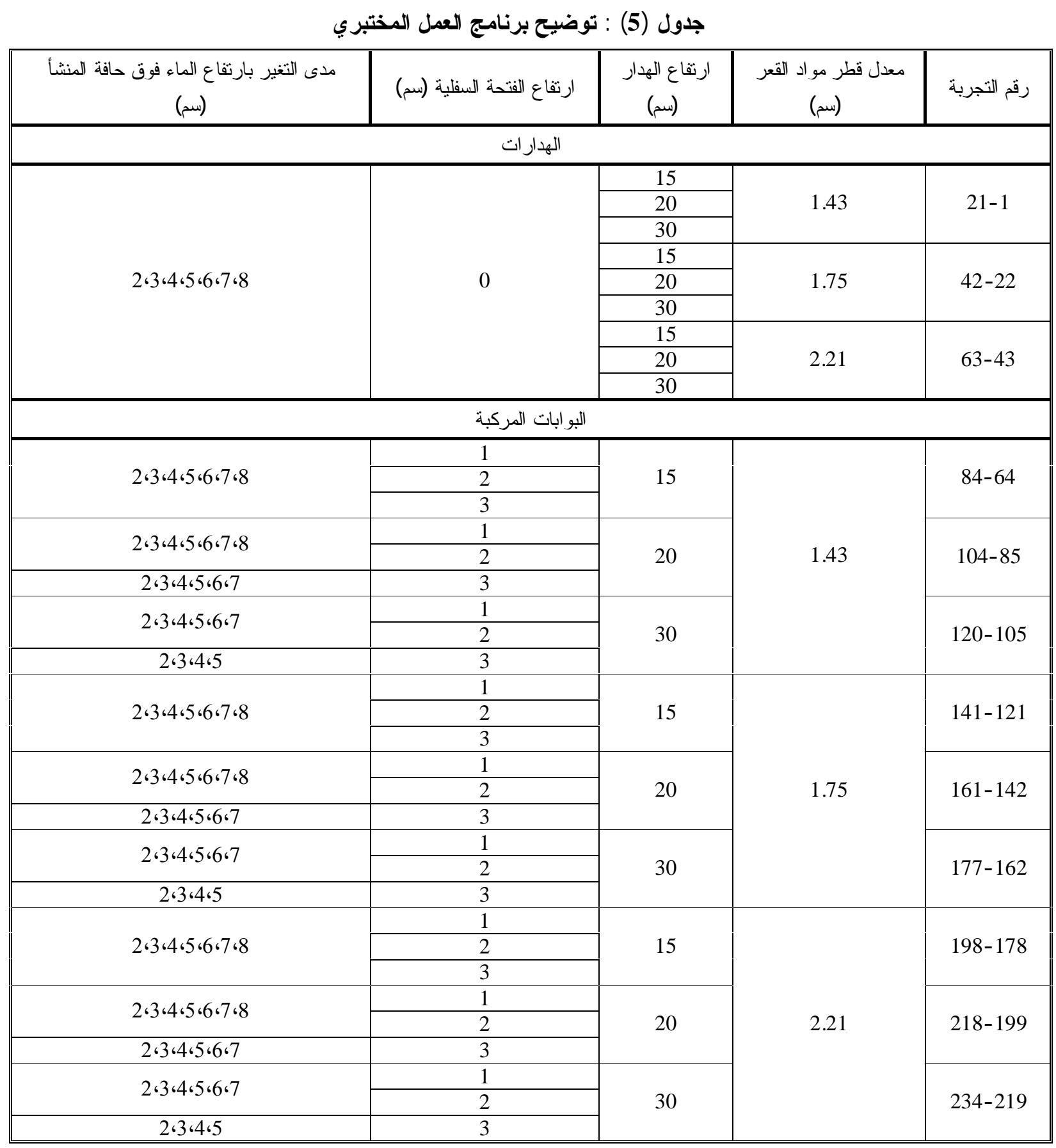

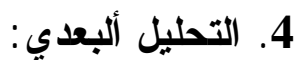

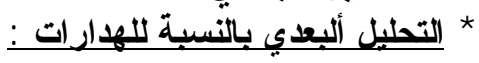

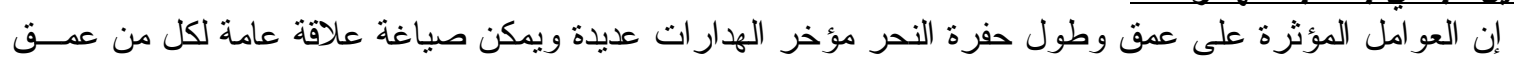

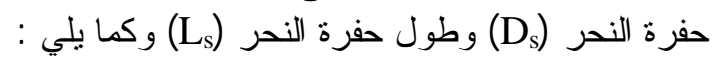


$D_{s}, L_{s}=f\left(q_{w}, D_{50}, P, \Delta H_{w}, g, \Delta \rho_{s}, \rho_{w}, \mu\right)$

وباستخدام التحليل ألبعدي بطريقة (П-theorem) للمتغير ات في المعادلة (1) نم التوصل إلى العلاقة اللابعدية التالية بالنسبة $\frac{D_{s}}{D_{50}}, \frac{L_{s}}{D_{50}}=f\left(\frac{\Delta H_{w}}{P}, F r 0, \operatorname{Re}\right)$

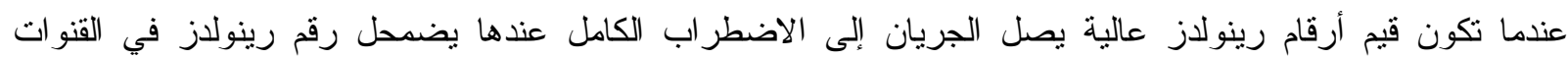

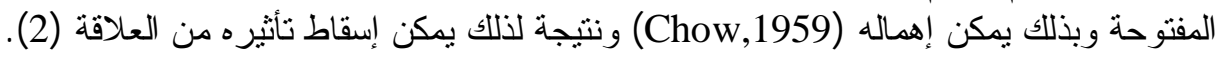

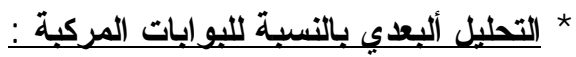

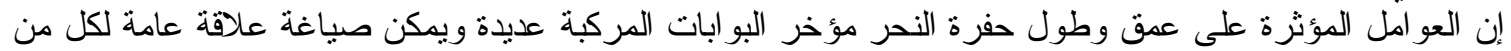
$D_{s}, L_{s}=f\left(q_{t}, q_{u}, q_{d}, D_{50}, P, \Delta H_{t}, h_{0}, \Delta \rho_{s}, \rho_{w}, \mu\right)$

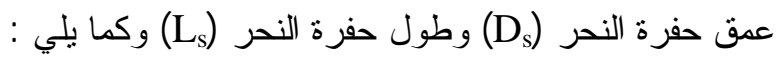

وباستخدام التحليل ألبعدي بطريقة (П-theorem) للمتغير ات في المعادلة (3) نم التوصل إلى العلاقـــة اللابعديــة

$\frac{D_{s}}{D_{50}}, \frac{L_{s}}{D_{50}}=f\left(\frac{\Delta H_{t}}{P}, \frac{h_{0}}{\Delta H_{t}}, \frac{q_{u}}{q_{d}}, F r 0, \mathrm{Re}\right)$

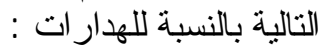

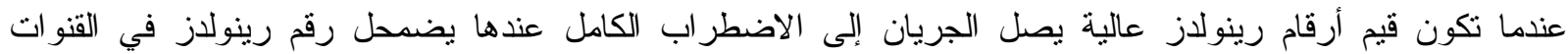

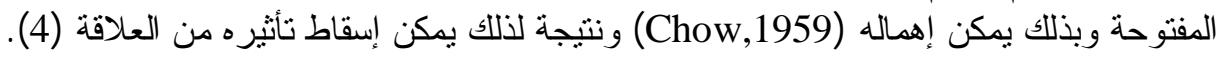

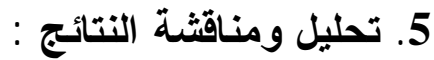
العلاقة بين العمق النسبي لحفرة النحر (

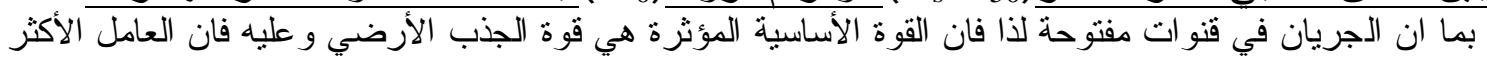
نأثثير أ على الجريان هو رقم فرود وكما موضح بالعلاقة التالية: $\frac{D_{s}}{D_{50}}=a_{1}+b_{1} \times$ Fro

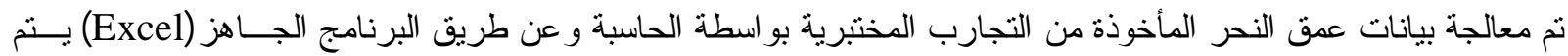

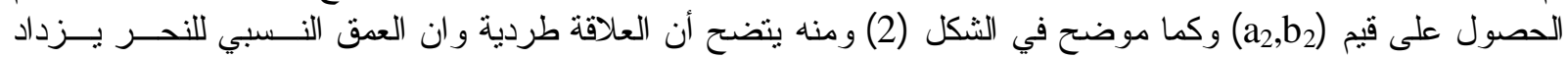

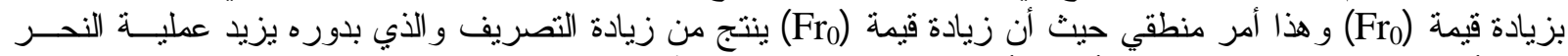

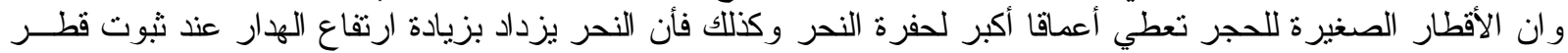

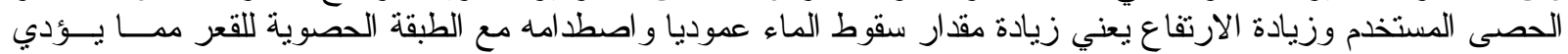

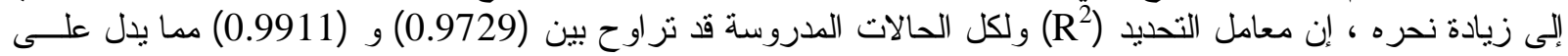
علاقات طردية جيدة.

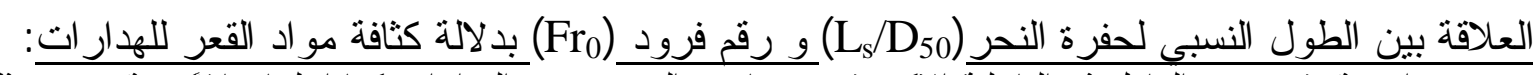

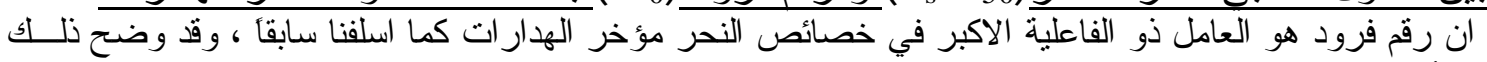

في المعادلة أدناه :

$\frac{L_{s}}{D_{50}}=a_{2}+b_{2} \times F r_{0}$

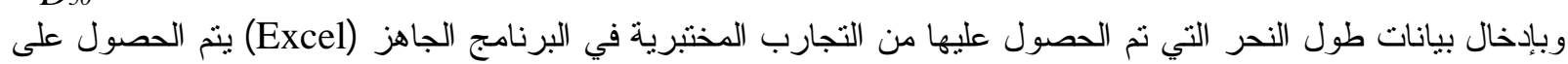
قيم 


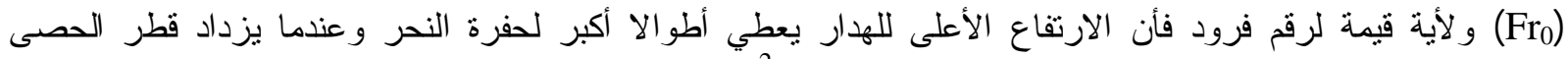

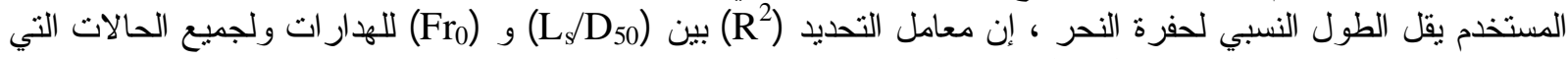

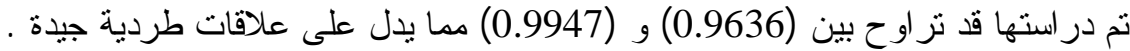

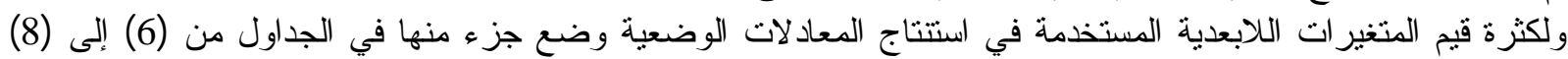

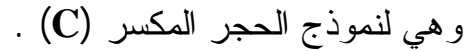

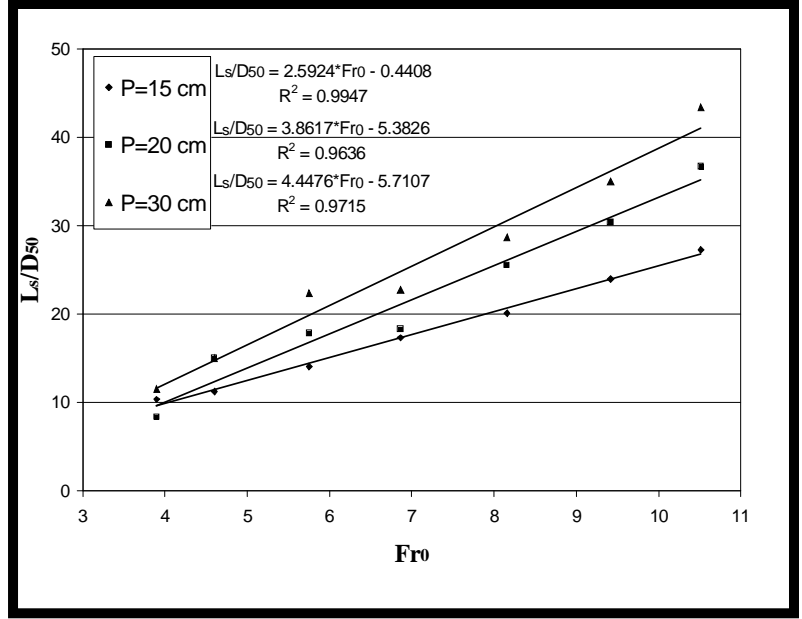

شكل (3) : العلاقة بين (A) (

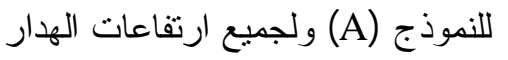

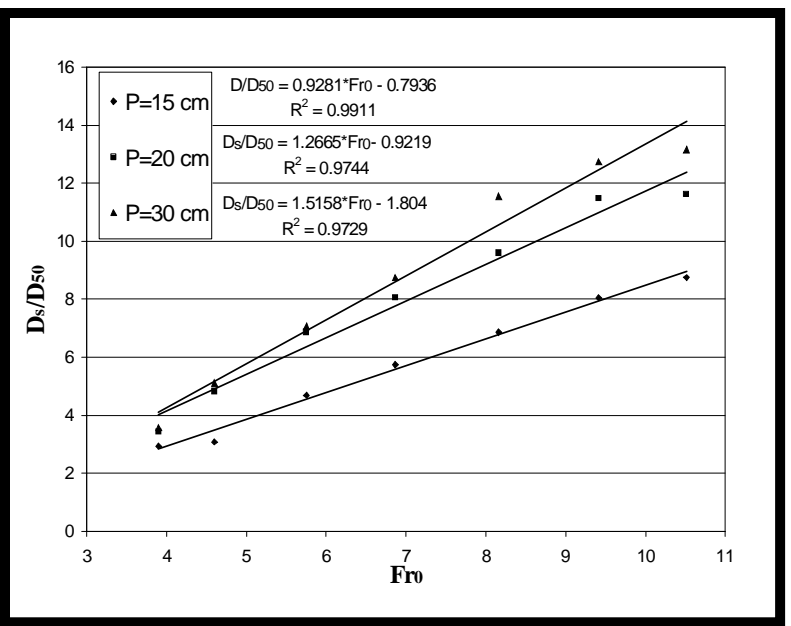

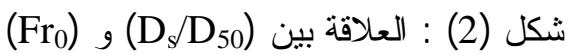

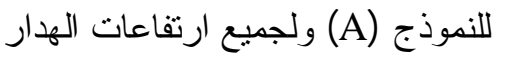

جدول (6): حسابات المتفيرات اللابعدية للهار بالارتفاع الأول (P) مع النموذج)

\begin{tabular}{|c|c|c|c|c|c|}
\hline $\mathbf{h}^{\prime}(\mathrm{cm})$ & $\mathbf{D}_{\mathbf{s}} / \mathbf{D}_{\mathbf{5 0}}$ & $\mathbf{F r}_{\mathbf{0}}$ & $\mathbf{h}_{\mathbf{0}} / \mathbf{\Delta} \mathbf{H}_{\mathbf{w}}$ & $\mathbf{L}_{\mathbf{s}} / \mathbf{D}_{\mathbf{5 0}}$ & $\boldsymbol{\Delta}_{\mathbf{w}} / \mathbf{P}$ \\
\hline $\mathbf{2}$ & 0.95130 & 2.23560 & 0.00000 & 4.75651 & 0.93333 \\
\hline $\mathbf{3}$ & 1.58550 & 2.63725 & 0.00000 & 5.93431 & 0.94667 \\
\hline $\mathbf{4}$ & 2.12911 & 3.29657 & 0.00000 & 8.06342 & 0.96667 \\
\hline $\mathbf{5}$ & 2.49151 & 3.93314 & 0.00000 & 9.87542 & 0.99333 \\
\hline $\mathbf{6}$ & 3.30691 & 4.67582 & 0.00000 & 11.46093 & 1.00676 \\
\hline $\mathbf{7}$ & 3.66931 & 5.39576 & 0.00000 & 13.04643 & 1.06000 \\
\hline $\mathbf{8}$ & 4.07701 & 6.02476 & 0.00000 & 14.63194 & 1.08667 \\
\hline
\end{tabular}

جدول (7): حسابات المتغيرات اللابعدية للهار بالارتفاع الثاني (C) مع النموذج

\begin{tabular}{|c|c|c|c|c|c|}
\hline $\mathbf{h}^{\prime}(\mathrm{cm})$ & $\mathbf{D}_{\mathbf{s}} / \mathbf{D}_{\mathbf{5 0}}$ & $\mathbf{F r}_{\mathbf{0}}$ & $\mathbf{h}_{\mathbf{0}} / \mathbf{\Delta H}_{\mathbf{w}}$ & $\mathbf{L}_{\mathbf{s}} / \mathbf{D}_{\mathbf{5 0}}$ & $\boldsymbol{\Delta}_{\mathbf{w}} / \mathbf{P}$ \\
\hline $\mathbf{2}$ & 1.08720 & 2.23560 & 0.00000 & 1.90260 & 0.95000 \\
\hline $\mathbf{3}$ & 1.63080 & 2.63725 & 0.00000 & 6.07022 & 0.96000 \\
\hline $\mathbf{4}$ & 2.12911 & 3.29657 & 0.00000 & 9.60362 & 0.97500 \\
\hline $\mathbf{5}$ & 2.58211 & 3.93314 & 0.00000 & 11.64213 & 0.99500 \\
\hline $\mathbf{6}$ & 3.39751 & 4.67582 & 0.00000 & 12.59343 & 1.00900 \\
\hline $\mathbf{7}$ & 4.30351 & 5.39576 & 0.00000 & 14.63194 & 1.04500 \\
\hline $\mathbf{8}$ & 5.25481 & 6.02476 & 0.00000 & 16.35334 & 1.06500 \\
\hline
\end{tabular}


نوري : دراسة النحر في الأرضيات الحصوية مؤخر الهارات والبوابات المركبة

جدول (8): حسابات المتفيرات اللابعدية للهار بالارتفاع الثالث (P) مع النموذج (C)

\begin{tabular}{|c|c|c|c|c|c|}
\hline \hline $\mathbf{h}^{\prime}(\mathrm{cm})$ & $\mathbf{D}_{\mathbf{s}} / \mathbf{D}_{\mathbf{5 0}}$ & $\mathbf{F r}_{\mathbf{0}}$ & $\mathbf{h}_{\mathbf{0}} / \mathbf{\Delta H}_{\mathbf{w}}$ & $\mathbf{L}_{\mathbf{s}} / \mathbf{D}_{\mathbf{5 0}}$ & $\boldsymbol{\Delta H}_{\mathbf{w}} / \mathbf{P}$ \\
\hline $\mathbf{2}$ & 1.67610 & 2.23560 & 0.00000 & 6.16082 & 0.96667 \\
\hline $\mathbf{3}$ & 2.03851 & 2.63725 & 0.00000 & 8.60702 & 0.97333 \\
\hline $\mathbf{4}$ & 2.21971 & 3.29657 & 0.00000 & 10.05663 & 0.98333 \\
\hline $\mathbf{5}$ & 3.12571 & 3.93314 & 0.00000 & 11.09853 & 0.99667 \\
\hline $\mathbf{6}$ & 3.98641 & 4.67582 & 0.00000 & 13.31823 & 1.00333 \\
\hline $\mathbf{7}$ & 5.20951 & 5.39576 & 0.00000 & 17.39524 & 1.03000 \\
\hline $\mathbf{8}$ & 6.07022 & 6.02476 & 0.00000 & 19.52435 & 1.04333 \\
\hline
\end{tabular}

علاقات وضعية لحساب عمق وطول النحر النسبي للهارات :

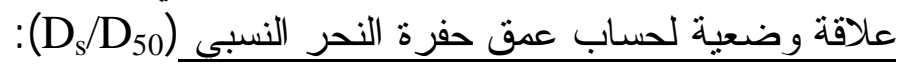

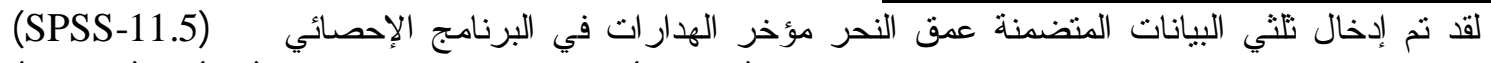
للحصول على علاقة وضعية لحساب العمق النسبي لحفرة النحر (D)

$\frac{D_{s}}{D_{50}}=0.971\left(F r_{0}\right)^{0.989}\left(\frac{\Delta H_{w}}{P}\right)^{0.291}$ وكما يأتي : لمالعى

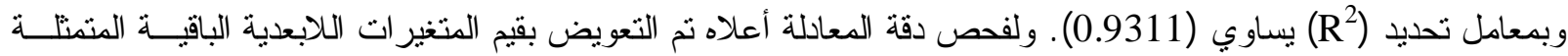
بكل من ( من التجارب (حيث أن هذه القيم المقاسة لم تستخدم في استتباط المعادلة (7) ، كما في الجدول (925) (9) حيث تر اوحت نسبة الفرق

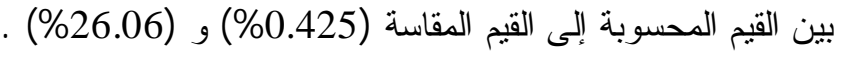

\begin{tabular}{|c|c|c|c|c|c|}
\hline \multicolumn{6}{|c|}{ المعادلة (7) للهدار ات } \\
\hline $\begin{array}{c}\mathrm{P} \\
(\mathrm{cm})\end{array}$ & $\begin{array}{l}\mathrm{D}_{50} \\
(\mathrm{~cm})\end{array}$ & $\begin{array}{c}\mathrm{h}^{\prime} \\
(\mathrm{cm})\end{array}$ & $\begin{array}{c}\mathrm{D}_{\mathrm{s}} / \mathrm{D}_{50} \\
\text { (Measured) }\end{array}$ & $\begin{array}{c}\mathrm{D}_{\mathrm{s}} / \mathrm{D}_{50} \\
\text { (Calculated) }\end{array}$ & نسبة الاختلاف بينهما \\
\hline \multirow{3}{*}{15} & 1.4285 & 7 & 8.050403 & 9.976885 & 19.30946 \\
\hline & 1.746 & 5 & 4.352806 & 5.317105 & 18.13578 \\
\hline & 2.2075 & 6 & 3.306908 & 4.472598 & 26.06292 \\
\hline \multirow{3}{*}{20} & 1.4285 & 3 & 4.830242 & 4.775194 & 1.152773 \\
\hline & 1.746 & 3 & 3.321879 & 3.545547 & 6.308436 \\
\hline & 2.2075 & 8 & 5.254813 & 5.841686 & 10.04629 \\
\hline \multirow{3}{*}{30} & 1.4285 & 2 & 3.570179 & 4.063481 & 12.13989 \\
\hline & 1.746 & 2 & 2.806415 & 3.017105 & 6.983192 \\
\hline & 2.2075 & 7 & 5.209513 & 5.18746 & 0.425128 \\
\hline
\end{tabular}




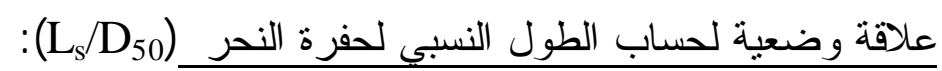

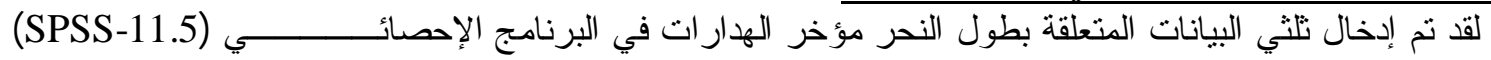

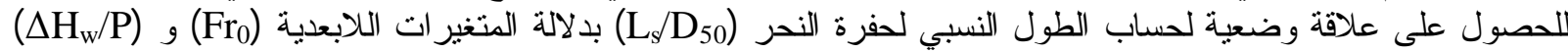
$\frac{L_{s}}{D_{50}}=4.114(F r o)^{0.825}\left(\frac{\Delta H_{w}}{P}\right)^{0.326}$ وكما بأتي :

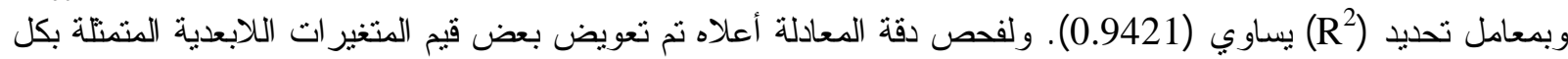

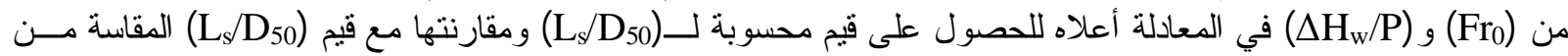

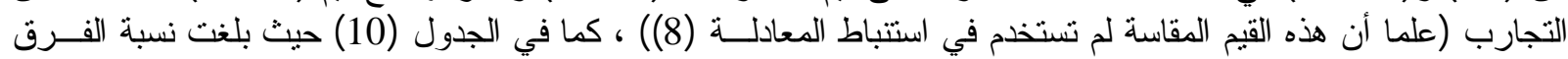

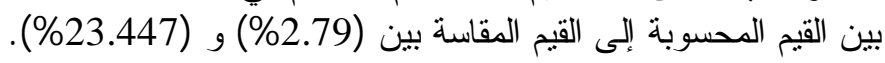

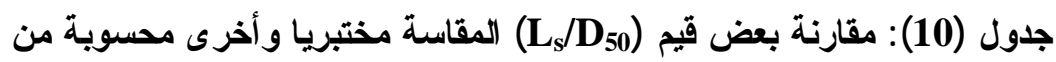

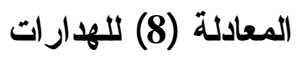

\begin{tabular}{|c|c|c|c|c|c|}
\hline $\begin{array}{c}\mathrm{P} \\
(\mathrm{cm})\end{array}$ & $\begin{array}{l}\mathrm{D}_{50} \\
(\mathrm{~cm})\end{array}$ & $\begin{array}{l}\mathrm{h} \\
(\mathrm{cm})\end{array}$ & $\begin{array}{c}\mathrm{D}_{\mathrm{s}} / \mathrm{D}_{50} \\
\text { (Measured) }\end{array}$ & $\begin{array}{c}\mathrm{D}_{f} / \mathrm{D}_{50} \\
\text { (Calculated) }\end{array}$ & نسبة الاختلاف بينهما \\
\hline \multirow{3}{*}{15} & 1.4285 & 7 & 24.0112 & 28.8649 & 16.81524 \\
\hline & 1.746 & 5 & 13.00115 & 16.9834 & 23.4479 \\
\hline & 2.2075 & 6 & 11.46093 & 14.71806 & 22.13019 \\
\hline \multirow{3}{*}{20} & 1.4285 & 3 & 15.05075 & 15.48274 & 2.790094 \\
\hline & 1.746 & 3 & 10.02291 & 12.07764 & 17.01271 \\
\hline & 2.2075 & 8 & 16.35334 & 18.47696 & 11.49332 \\
\hline \multirow{3}{*}{30} & 1.4285 & 2 & 11.55058 & 13.5403 & 14.69481 \\
\hline & 1.746 & 2 & 8.476518 & 10.56241 & 19.74822 \\
\hline & 2.2075 & 7 & 17.39524 & 16.68755 & 4.240868 \\
\hline
\end{tabular}

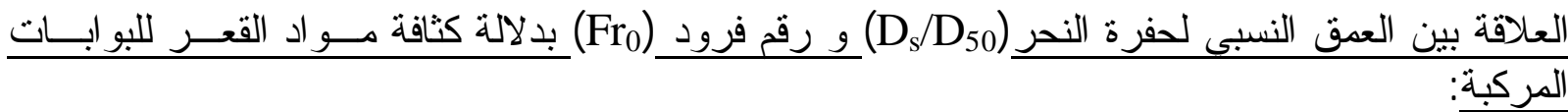
إن القنوات المفتوحة تتأثر بشكل كبير بالجاذبية الأرضية وبالثالي فأن رقم فرود هو الأكثر نأثير أ وكما يلي : $\frac{D_{s}}{D_{50}}=a_{3}+b_{3} \times F r_{0}$

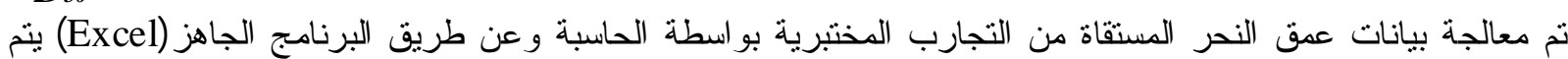

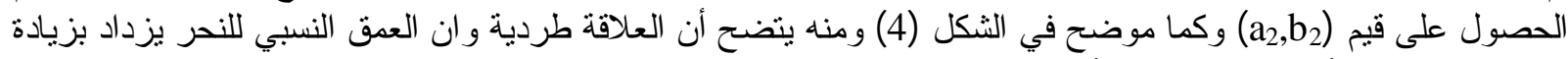
قيمة

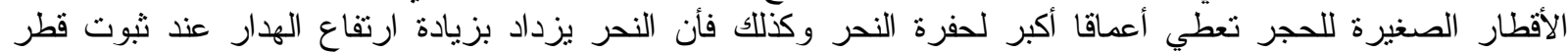

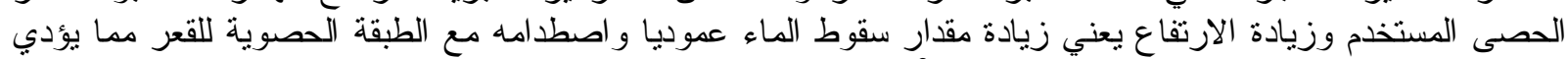

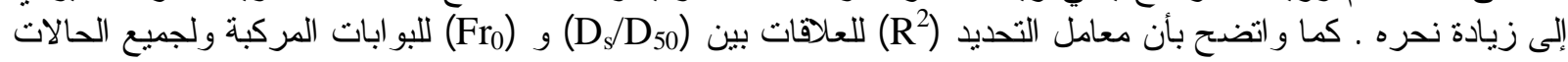

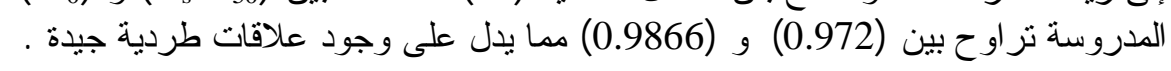




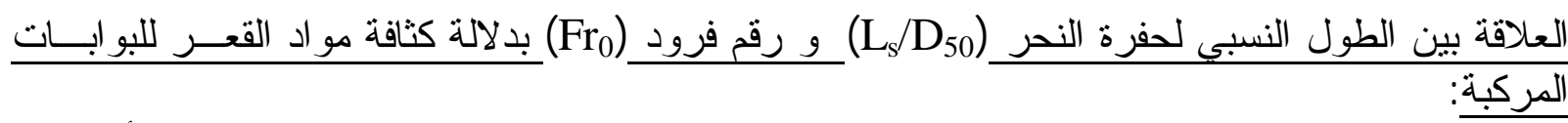

رقم فرود هو العامل المؤثر على النحر مؤخر البوابات المركبة ، لذا تم تحليل البيانات المختبرية ووجد بأن العبان العلاقة الخطية هي أفضل العلاقات التي تربط بين (Lئ

$\frac{L_{s}}{D_{50}}=a_{4}+b_{4} \times$ Fro

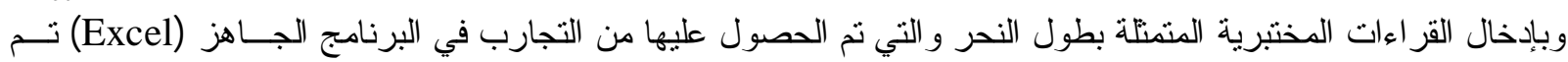

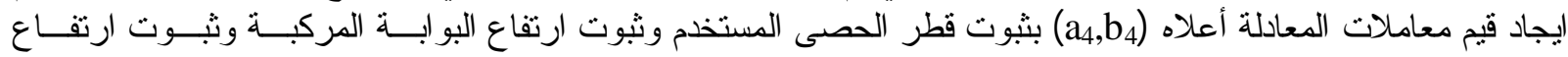

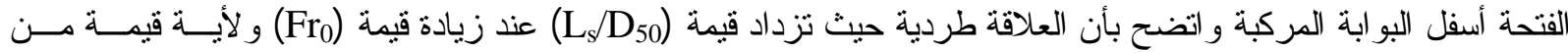
(أبro

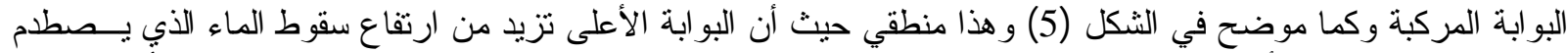

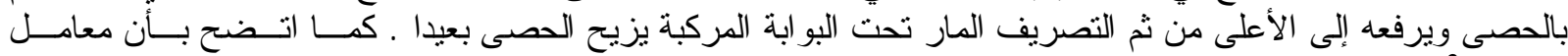

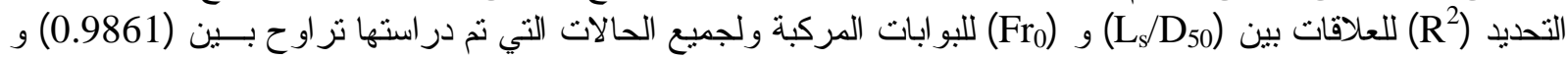

(0.9985) مما بدل على علاقات طردية جيدة.

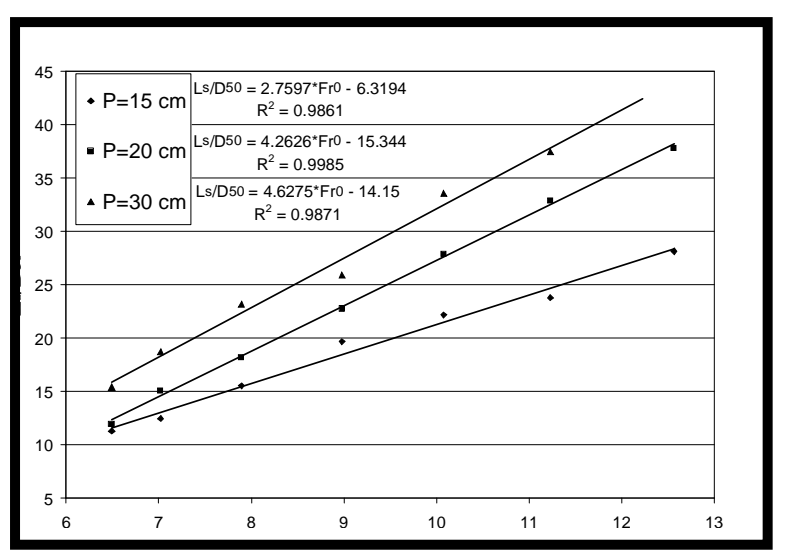

شكل (5) : العلاقة بين (

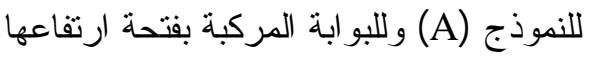
ولجميع الارتفاعات

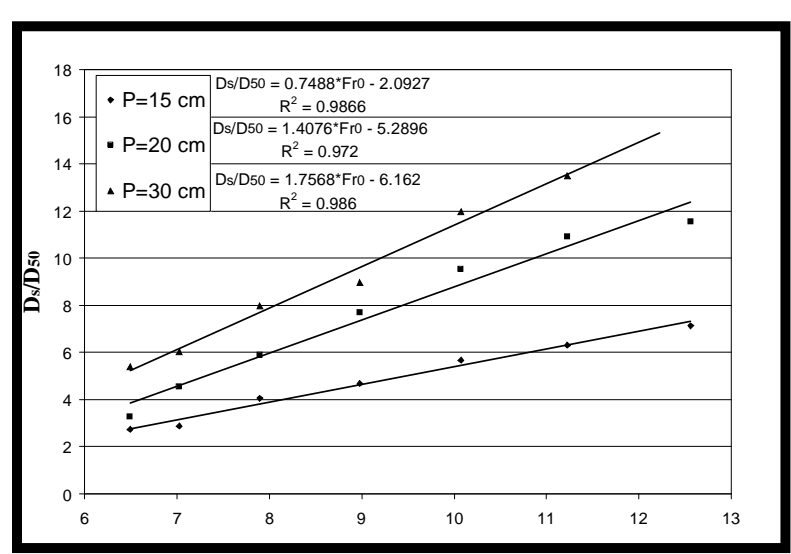

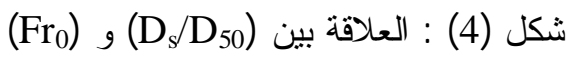
للنموذج (A) وللبو ابة المركبة بفتحة ارتفاعها ولجميع الارتفاعات (holcm)

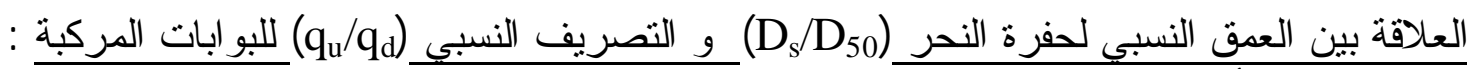

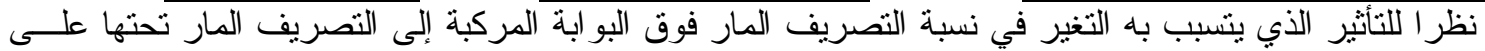

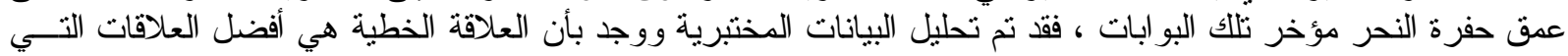

$$
\frac{D_{s}}{D_{50}}=a_{5}+b_{5} \times \frac{q_{u}}{q_{d}}
$$

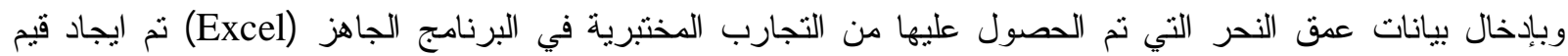

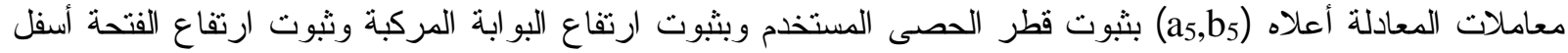

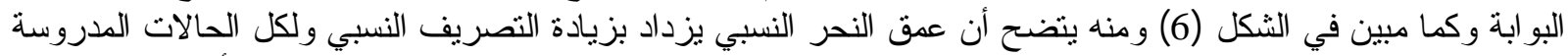

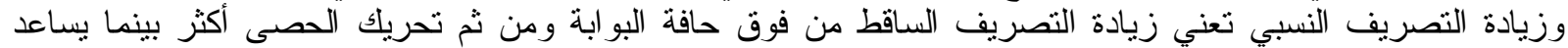

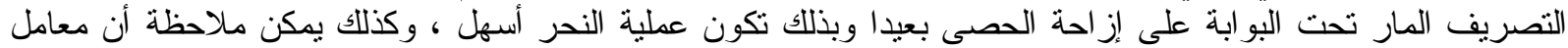
التحديد (R) عال في هذه الأشكال مما يعني أن العلاقة قوية وان التصريف النسبي له تأثير كبير على العمق النسبي لحفرة 


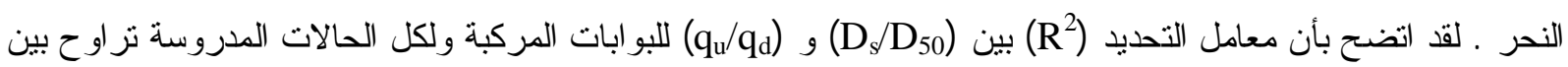

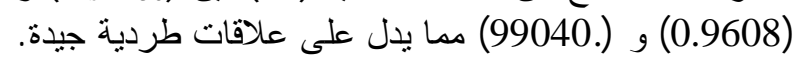

العلاقة بين الطول النسبي لحفرة النحر (L)/

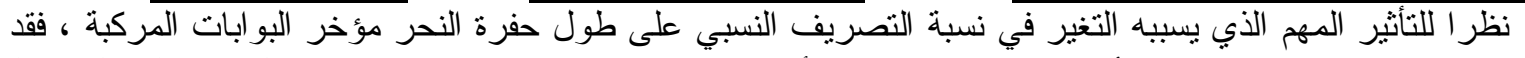

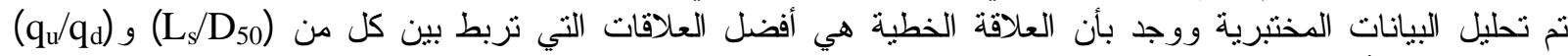

$\frac{L_{s}}{D_{50}}=a_{6}+b_{6} \times \frac{q_{u}}{q_{d}}$ و الموضحة في أدناه :

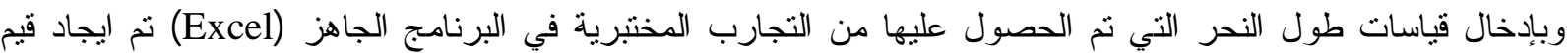

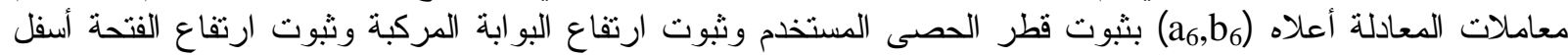

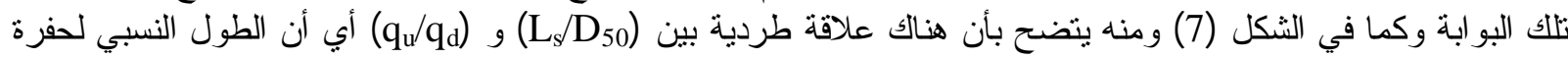

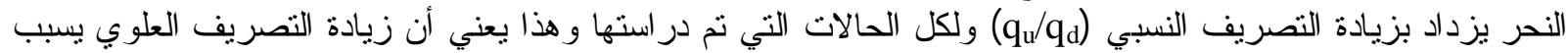

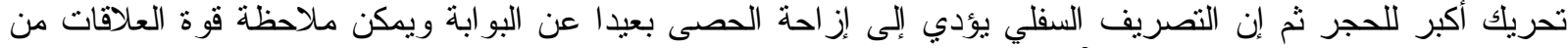

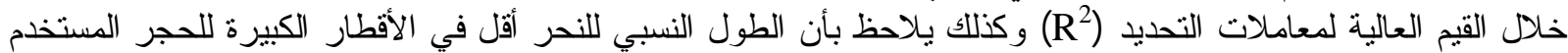

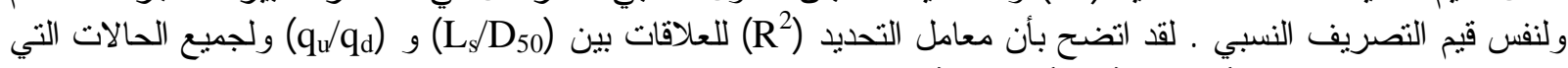

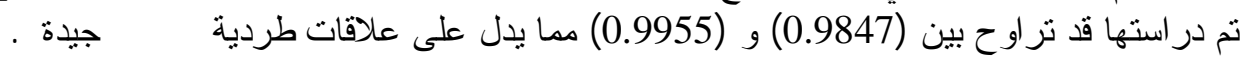

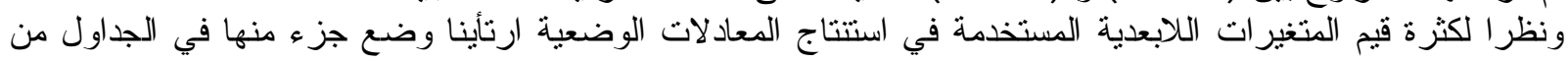

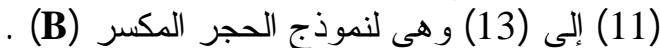
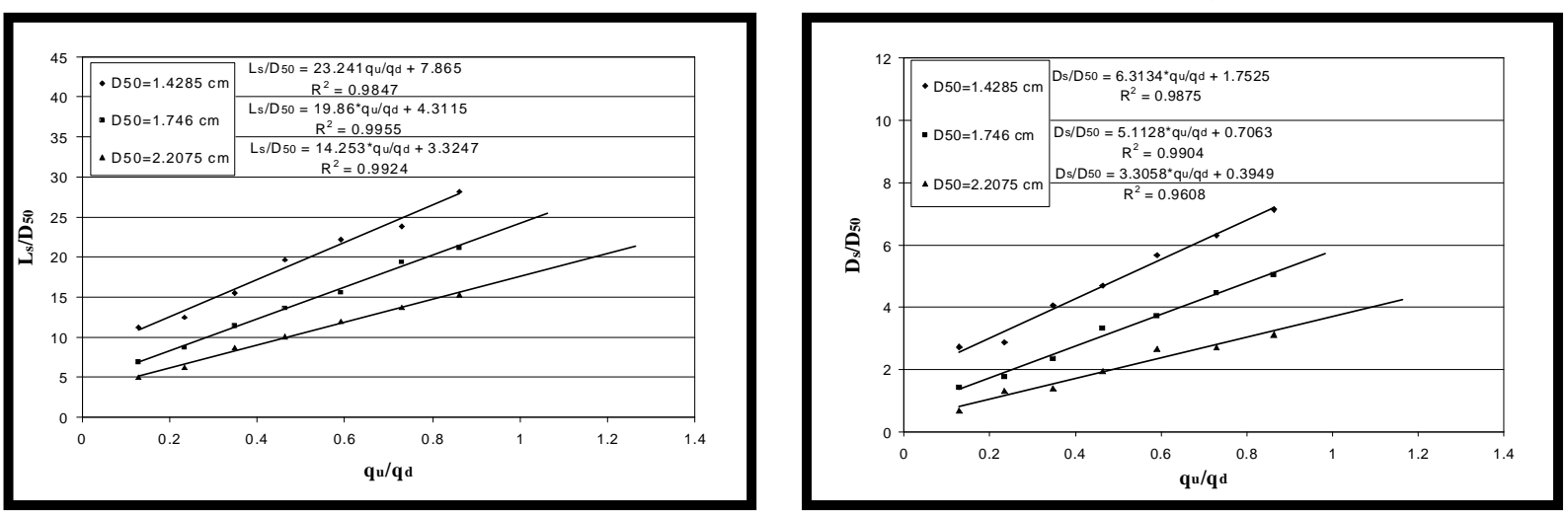

شكل (7) : العلاقة بين ( (

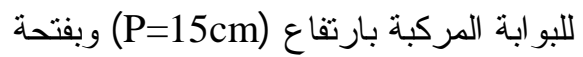
ارتفاعها (h=1cm) ولجميع نماذج مو اد

$$
\begin{aligned}
& \text { شكل (6) : العلاقة بين ( } \\
& \text { للبو ابة المركبة بارتفاع (6=15cm) وبفتحة }
\end{aligned}
$$

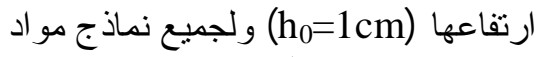

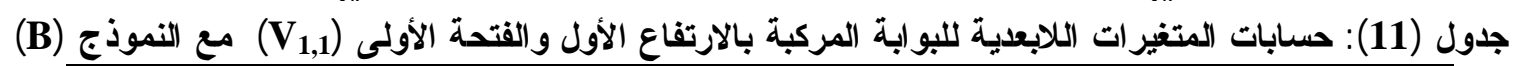

\begin{tabular}{|c|c|c|c|c|c|c|}
\hline $\mathbf{h}^{\prime}(\mathrm{cm})$ & $\mathbf{D}_{\mathbf{s}} / \mathbf{D}_{\mathbf{5 0}}$ & $\mathbf{F r}_{\mathbf{0}}$ & $\mathbf{h}_{\mathbf{0}} / \mathbf{\Delta H}_{\mathbf{t}}$ & $\mathbf{L}_{\mathbf{s}} / \mathbf{D}_{\mathbf{5 0}}$ & $\mathbf{q}_{\mathbf{u}} / \mathbf{q}_{\mathbf{d}}$ & $\Delta \mathbf{H}_{\mathbf{t}} / \mathbf{P}$ \\
\hline $\mathbf{2}$ & 1.43184 & 5.28979 & 0.0840 & 6.93013 & 0.1287 & 0.79333 \\
\hline $\mathbf{3}$ & 1.77549 & 5.72073 & 0.0833 & 8.70561 & 0.2343 & 0.80000 \\
\hline $\mathbf{4}$ & 2.34822 & 6.43178 & 0.0826 & 11.45475 & 0.3476 & 0.80667 \\
\hline $\mathbf{5}$ & 3.32188 & 7.31521 & 0.0826 & 13.63116 & 0.4637 & 0.80667 \\
\hline $\mathbf{6}$ & 3.72279 & 8.20941 & 0.0813 & 15.57847 & 0.5908 & 0.82000 \\
\hline $\mathbf{7}$ & 4.46735 & 9.14671 & 0.0819 & 19.35853 & 0.7291 & 0.81333 \\
\hline $\mathbf{8}$ & 5.04009 & 10.23483 & 0.0806 & 21.19129 & 0.8628 & 0.82667 \\
\hline
\end{tabular}


نوري : دراسة النحر في الأرضيات الحصوية مؤخر الهارات والبوابات المركبة

جلول (12): حسابات المتغيرات اللابعدية للبوابة المركبة بالارتفاع الثاني و الفتحة الأولى (V) (B) مع النموذج (12)

\begin{tabular}{|c|c|c|c|c|c|c|}
\hline $\mathbf{h}^{\prime}(\mathrm{cm})$ & $\mathbf{D}_{\mathbf{s}} / \mathbf{D}_{\mathbf{5 0}}$ & $\mathbf{F r}_{\mathbf{0}}$ & $\mathbf{h}_{\mathbf{0}} / \mathbf{\Delta} \mathbf{H}_{\mathbf{t}}$ & $\mathbf{L}_{\mathbf{s}} / \mathbf{D}_{\mathbf{5 0}}$ & $\mathbf{q}_{\mathbf{u}} / \mathbf{q}_{\mathbf{d}}$ & $\Delta \mathbf{H}_{\mathbf{t}} / \mathbf{P}$ \\
\hline \hline $\mathbf{2}$ & 1.83276 & 5.28979 & 0.0591 & 9.04926 & 0.1287 & 0.80476 \\
\hline $\mathbf{3}$ & 2.34822 & 5.72073 & 0.0588 & 10.13746 & 0.2320 & 0.80952 \\
\hline $\mathbf{4}$ & 3.37915 & 6.43178 & 0.5848 & 13.63116 & 0.3446 & 0.81429 \\
\hline $\mathbf{5}$ & 3.55097 & 7.31521 & 0.5848 & 15.34937 & 0.4571 & 0.81429 \\
\hline $\mathbf{6}$ & 4.69645 & 8.20941 & 0.0578 & 18.55670 & 0.5809 & 0.82381 \\
\hline $\mathbf{7}$ & 5.61283 & 9.14671 & 0.0581 & 21.24857 & 0.7117 & 0.81905 \\
\hline $\mathbf{8}$ & 6.70103 & 10.23483 & 0.0575 & 26.63230 & 0.8375 & 0.82857 \\
\hline
\end{tabular}

جدول (13): حسابات المتغير ات اللابعدية للبو ابة المركبة بالارتفاع الثالث و الفتحة الأولى (V1,3) مع النموذج (B)

\begin{tabular}{||c|c|c|c|c|c|c||}
\hline $\mathbf{h}^{\prime}(\mathrm{cm})$ & $\mathbf{D}_{\boldsymbol{s}} / \mathbf{D}_{\mathbf{5 0}}$ & $\mathbf{F r}_{\mathbf{0}}$ & $\mathbf{h}_{\mathbf{0}} / \mathbf{\Delta} \mathbf{H}_{\mathbf{t}}$ & $\mathbf{L}_{\boldsymbol{s}} / \mathbf{D}_{\mathbf{5 0}}$ & $\mathbf{q}_{\mathbf{u}} / \mathbf{q}_{\mathbf{d}}$ & $\mathbf{\Delta H}_{\mathbf{t}} / \mathbf{P}$ \\
\hline \hline $\mathbf{2}$ & 4.35281 & 5.28979 & 0.03717 & 9.45017 & 0.1261 & 0.86774 \\
\hline $\mathbf{3}$ & 4.75372 & 5.72073 & 0.03704 & 12.60023 & 0.2992 & 0.87097 \\
\hline $\mathbf{4}$ & 4.86827 & 6.43178 & 0.03690 & 15.06300 & 0.3386 & 0.87419 \\
\hline $\mathbf{5}$ & 6.30011 & 7.31521 & 0.03690 & 19.47308 & 0.4508 & 0.87419 \\
\hline $\mathbf{6}$ & 7.33104 & 8.20941 & 0.03663 & 23.76861 & 0.5679 & 0.88065 \\
\hline $\mathbf{7}$ & 9.27835 & 9.14671 & 0.03676 & 28.06415 & 0.6946 & 0.87742 \\
\hline $\mathbf{8}$ & - & - & - & - & - & - \\
\hline
\end{tabular}

علاقات وضعية لحسب عمق وطول النحر النسبي للبوابات المركبة:

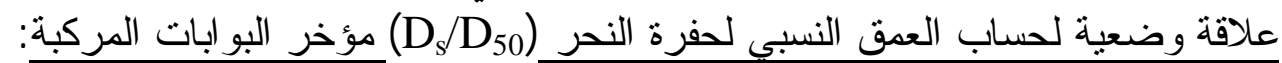

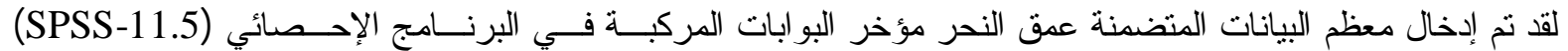

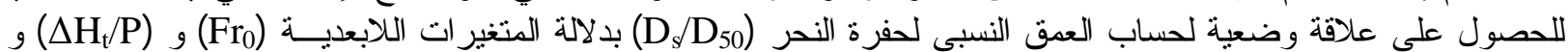

$$
\frac{D_{s}}{D_{50}}=1.421 \frac{(F r 0)^{0.751}\left(\frac{\Delta H_{t}}{P}\right)^{0.451}\left(\frac{q_{u}}{q_{d}}\right)^{0.517}}{\left(\frac{h_{o}}{\Delta H_{t}}\right)^{0.122}}
$$$$
\text { : وكما يأتي }\left(\mathrm{q}_{\mathrm{u}} / \mathrm{q}_{\mathrm{d}}\right) \text { و }\left(\mathrm{h}_{0} / \Delta \mathrm{H}_{\mathrm{t}}\right)
$$

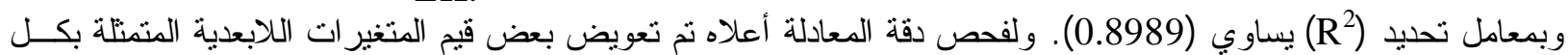
من (

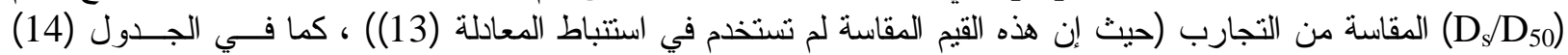

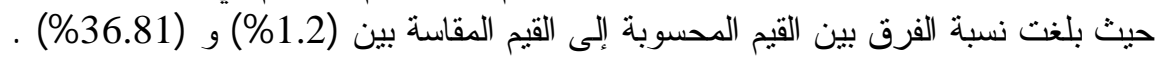

علاقة وضعية لحساب الطول النسبي لحفرة النحر (Ls/

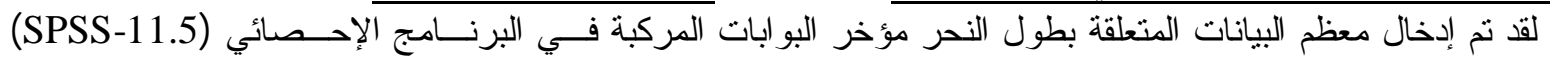

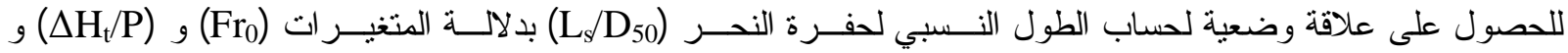

$$
\frac{L_{s}}{D_{50}}=3.1232 \frac{\left(F r_{0}\right)^{0.899}\left(\frac{\Delta H_{t}}{P}\right)^{0.246}\left(\frac{q_{u}}{q_{d}}\right)^{0.621}}{\left(\frac{h_{o}}{\Delta H_{t}}\right)^{0.171}}
$$


جدول (14): مقارنة بعض قيم (Ds/D) (المقاسة مختبريا و أخرى محسوبة من

المعادلة (13) للبو ابات المركبة

\begin{tabular}{|c|c|c|c|c|c|c|}
\hline $\begin{array}{c}\mathbf{P} \\
(\mathrm{cm}) \\
\end{array}$ & $\begin{array}{c}\mathbf{h}_{\mathbf{0}} \\
(\mathrm{cm}) \\
\end{array}$ & $\begin{array}{c}\mathbf{D}_{\mathbf{5 0}} \\
(\mathrm{cm}) \\
\end{array}$ & $\begin{array}{c}\mathrm{h} \\
(\mathrm{cm}) \\
\end{array}$ & $\begin{array}{c}\mathbf{D}_{\mathbf{s}} / \mathbf{D}_{\mathbf{5 0}} \\
\text { (Measured) } \\
\end{array}$ & $\begin{array}{c}\mathbf{D}_{\mathbf{s}} / \mathbf{D}_{\mathbf{5 0}} \\
\text { (Calculated) } \\
\end{array}$ & نسبة الاختلاف بينهما \\
\hline \multirow{4}{*}{15} & 3 & 1.4285 & 5 & 5.6003 & 5.668 & 1.2 \\
\hline & 2 & 1.746 & 4 & 2.7491 & 3.802 & 27.69 \\
\hline & 3 & 1.746 & 4 & 3.3219 & 3.629 & 8.465 \\
\hline & 2 & 2.2075 & 7 & 11.761 & 9.084 & 36.81 \\
\hline \multirow{8}{*}{20} & 3 & 1.4285 & 7 & 12.741 & 10.11 & 26.04 \\
\hline & 1 & 1.746 & 2 & 1.8328 & 2.285 & 19.77 \\
\hline & 2 & 1.746 & 4 & 4.3528 & 4.092 & 6.362 \\
\hline & 3 & 1.746 & 6 & 7.4456 & 6.794 & 9.597 \\
\hline & 2 & 2.2075 & 3 & 2.3103 & 2.355 & 1.914 \\
\hline & 3 & 2.2075 & 4 & 3.3069 & 3.438 & 3.808 \\
\hline & 1 & 1.4285 & 5 & 8.9604 & 7.049 & 9.24 \\
\hline & 2 & 1.4285 & 7 & 14.771 & 11.4 & 12.39 \\
\hline \multirow{4}{*}{30} & 1 & 1.746 & 5 & 6.3001 & 6.092 & 3.411 \\
\hline & 2 & 1.746 & 7 & 9.8511 & 8.096 & 21.68 \\
\hline & 1 & 2.2075 & 2 & 2.0838 & 1.876 & 11.09 \\
\hline & 2 & 2.2075 & 4 & 3.8505 & 3.352 & 14.88 \\
\hline
\end{tabular}

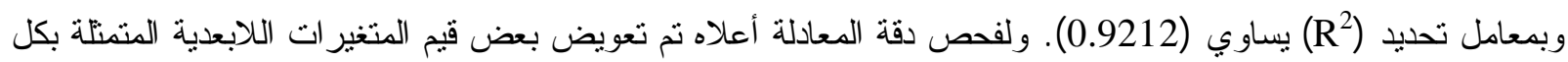

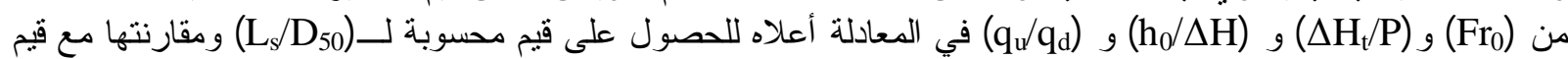

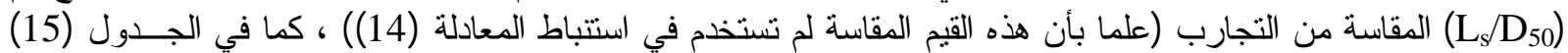

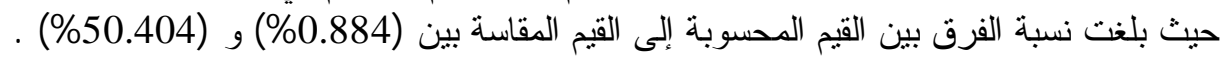

جدول (15): مقارنة بعض قيم (Ls/ $)$ (المقاسة مختبريا و أخرى محسوبة من المعادلة (14) للبو ابات المركبة المقاسية

\begin{tabular}{|c|c|c|c|c|c|c|}
\hline $\begin{array}{c}\mathbf{P} \\
(\mathrm{cm})\end{array}$ & $\begin{array}{c}\mathbf{h}_{\mathbf{0}} \\
(\mathrm{cm})\end{array}$ & $\begin{array}{l}\mathbf{D}_{50} \\
(\mathrm{~cm})\end{array}$ & $\begin{array}{l}\mathrm{h}^{\prime} \\
(\mathrm{cm})\end{array}$ & $\begin{array}{c}\mathbf{L}_{\mathbf{s}} / \mathbf{D}_{\mathbf{5 0}} \\
\text { (Measured) }\end{array}$ & $\begin{array}{c}\mathbf{L}_{\mathbf{s}} / \mathbf{D}_{\mathbf{5 0}} \\
\text { (Calculated) }\end{array}$ & نسبة الاختلاف بينهما \\
\hline \multirow{5}{*}{15} & 3 & 1.4285 & 5 & 19.951 & 17.69805 & 12.72994 \\
\hline & 2 & 1.746 & 4 & 12.14204 & 11.0253 & 10.12888 \\
\hline & 3 & 1.746 & 4 & 13.68843 & 10.39327 & 31.70476 \\
\hline & 2 & 2.2075 & 3 & 6.387316 & 30.82877 & 20.29775 \\
\hline & 2 & 1.4285 & 7 & 37.17186 & 34.36942 & 12.22747 \\
\hline \multirow{7}{*}{20} & 3 & 1.4285 & 7 & 38.57193 & 6.016622 & 50.40425 \\
\hline & 2 & 1.746 & 4 & 14.20389 & 11.88 & 19.56143 \\
\hline & 3 & 1.746 & 6 & 23.76861 & 21.44184 & 10.85158 \\
\hline & 2 & 2.2075 & 4 & 9.286523 & 6.17484 & 6.375495 \\
\hline & 3 & 2.2075 & 4 & 9.739524 & 9.642811 & 1.002958 \\
\hline & 1 & 1.4285 & 5 & 25.9013 & 23.11044 & 1.55472 \\
\hline & 2 & 1.4285 & 7 & 39.55198 & 40.03916 & 5.776739 \\
\hline \multirow{4}{*}{30} & 1 & 1.746 & 5 & 19.47308 & 19.30245 & 0.883964 \\
\hline & 2 & 1.746 & 7 & 29.49599 & 26.78246 & 10.13176 \\
\hline & 1 & 2.2075 & 2 & 6.523216 & 4.769868 & 36.75884 \\
\hline & 2 & 2.2075 & 4 & 12.23103 & 9.389359 & 30.26481 \\
\hline
\end{tabular}


1. من التحليل ألبعدي اللعو امل المؤثرة على عمق وطول حفرة النحر مؤخر الهارات وات و البو ابات المركبة وبوابات الكسح

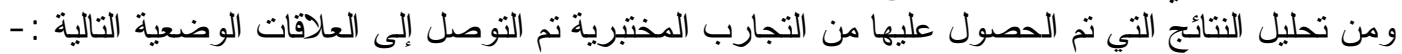

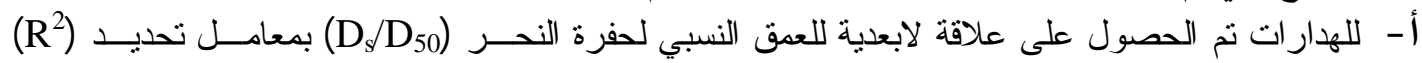

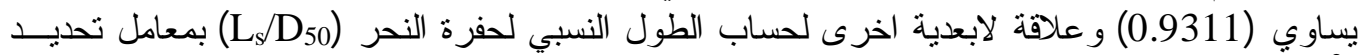

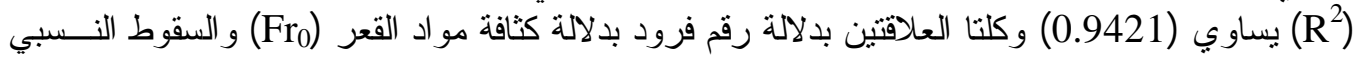

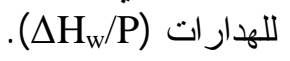

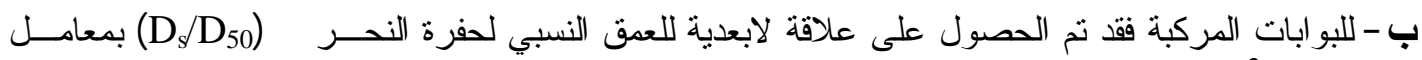

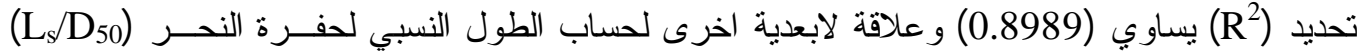

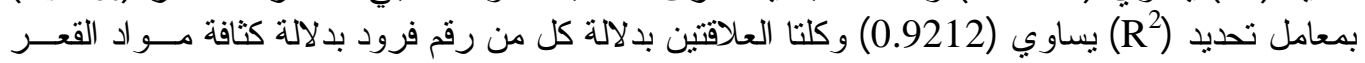

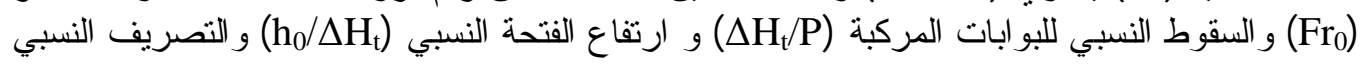
. $\left(\mathrm{q}_{\mathrm{u}} / \mathrm{q}_{\mathrm{d}}\right)$

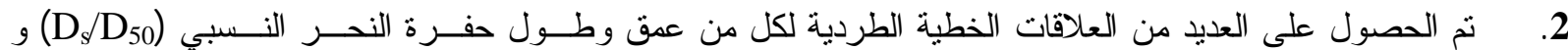
(Ls $\left.\mathrm{L}_{50}\right)$ التصريف النسبي (qu/

اتضح من التجارب الني أجريت هو أن عمق وطول حفرة النيات النحر عندما يمر الجريان أعلى وأسفل البوابة المركبة اقل

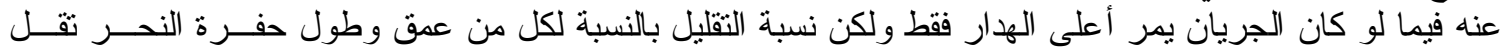

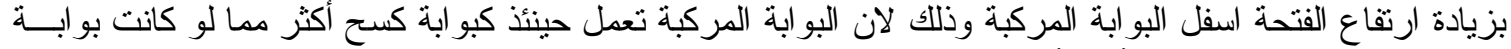

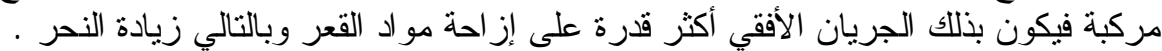

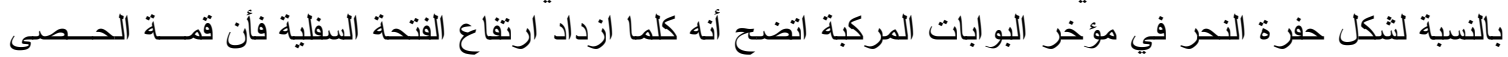

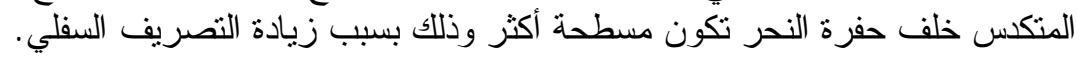

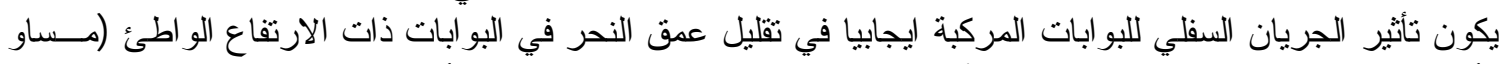

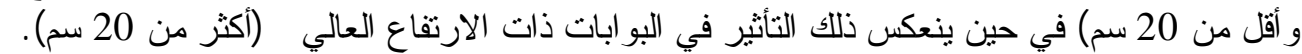

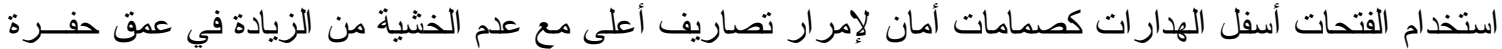

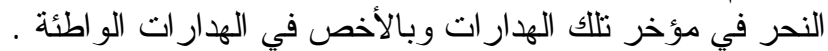

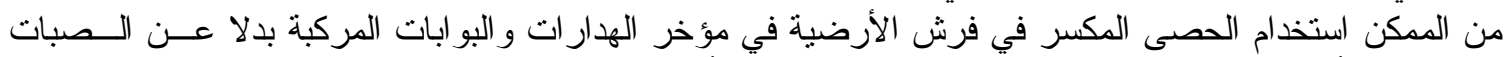
الخرسانية الأمر الذي يقلل من الكلفة الإقتصادية لتبطين تلأك الأرضيات.

1. الحافظ , إبراهيم عادل ، (2005) ـ " دراسة مختبرية للنحر في الأرضيات الحجرية مؤخر الهدار ات المتلثنية" . رسالة

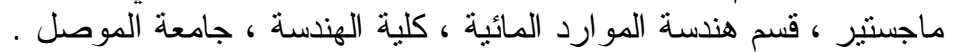

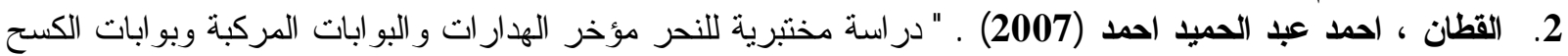

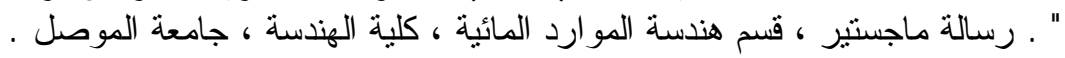

3. Asai, K. , Mimura, Y. and Kawamoto, N. (2002) . "Experimental Study on Scour and Sedimentation of River Bed by Overflow from Weir " . J. Hyd. Res., IAHR, Vol. 15, No. 1, pp. 45- 50 .

4. British Standard Institution (1965) . " Method of Measurement of Liquid Flow

Open Channel ". BS3680, part (A), London, England.

5. Chen, Z. , Shao, X. and Zhang, J. (2005) . " Experimental Study on the Upstream Water Level Rise and Downstream Scour Length of a Submerged Dam " . J. Hyd. Res., IAHR, Vol. 43, No. 6, pp. 703-709. 


\section{$\begin{array}{llll}\text { Al-Rafidain Engineering } & \text { Vol.17 } & \text { No.5 } & \text { October } 2009\end{array}$}

6. Chow, V. T. (1959) . "Open Channel Hydraulics " . Mac Graw -Hill International Company, International Student Edition .

7. Dargahi, B. (2003) . " Scour Development Downstream of Spillway " . J. Hyd. Res., IAHR, Vol. 41, No. 4, pp. 417-426.

8. Simons, D. and Şentürk, F. (1992) . " Sediment Transport Technology " .Water Resources Publications, Littleton, Colorado, U.S.A.

9. Uyumaz, A. (1988) . " Scour Downstream of Vertical Gate " . J . Hyd. Eng., ASCE, Vol. 114, No. 7, pp. 811- 816 .

$$
\text { تم اجراء البحث في كلية الهندسة - جامعة الموصل }
$$

